\title{
Enhancing Mobile Services with DVB-S2X Superframing
}

\author{
N. Mazzali ${ }^{1 *}$, S. Boumard ${ }^{2}$, J. Kinnunen ${ }^{3}$, B. Shankar M. R. ${ }^{1}$, M. Kiviranta ${ }^{2}$, and \\ N. Alagha ${ }^{4}$ \\ ${ }^{1}$ Interdisciplinary Centre for Security, Reliability and Trust (SnT), University of Luxembourg, 29, avenue J. F. Kennedy, \\ L-1855 Luxembourg, Luxembourg \\ ${ }^{2}$ VTT Technical Research Center of Finland Ltd, Kaitoväylä 1, FI-90570 Oulu, Finland \\ ${ }^{3}$ Bittium Corp., Tutkijantie 8, FI-90590 Oulu, Finland \\ ${ }^{4}$ European Space Research and Technology Centre (ESTEC), Keplerlaan 1, 2201 AZ Noordwijk, The Netherlands
}

\begin{abstract}
SUMMARY
DVB-S2X is the cornerstone for satellite communication standards forming the state-of-the-art of broadband satellite waveforms. In this paper we propose new application scenarios and advanced techniques, including a reference design implementing superframing, predistortion, a robust synchronization chain, and a plugand-play channel interleaver. We demonstrate by means of software simulations and hardware tests that the DVB-S2X can be a common technology enabler for land-mobile, aeronautical, and maritime satellite scenarios in addition to the more traditional VSAT scenario, even in very challenging conditions (e.g., very low SNR). Copyright (C) 2018 John Wiley \& Sons, Ltd.

Received ...
\end{abstract}

KEY WORDS: DVB-S2X; superframing; predistortion; channel interleaver; synchronization; hardware demonstrator

\section{INTRODUCTION}

The second generation of the digital video broadcasting standard for satellites (DVB-S2) [1] developed by the DVB Project in 2003 and ratified by the European Telecommunications Standards Institute (ETSI) in 2005 has been the cornerstone of satellite communication standards forming the basis of digital satellite television transmission across the globe. Based on a number of refinements and innovations over the DVB-S2, the extension to the standard (which includes sharper roll-offs, modulation and coding (MODCOD) upgrades, very low signal-to-noise ratio (SNR) paradigm, and superframing structure) has been formalized in 2014 in [2] under the name of DVB-S2X. This version represents the state-of-the-art of broadband satellite waveforms, and has been adopted as

\footnotetext{
*Correspondence to: Interdisciplinary Centre for Security, Reliability and Trust (SnT), University of Luxembourg, 29, avenue J. F. Kennedy, L-1855 Luxembourg, Luxembourg; email: nicolo.mazzali@uni.lu

Contract/grant sponsor: ESA-funded ARTES-5.1 activity "Novel Ground Components Prototype beyond DVB-S2 for Broadband Satellite Networks"; contract/grant number: 4000110120/14/NL/NR

Copyright (c) 2018 John Wiley \& Sons, Ltd.

Prepared using satauth.cls [Version: 2010/05/13 v2.00]
} 
starting point for the presented investigation. The biggest demand for the extensions of the DVBS2 standard came from video contribution and high-speed Internet protocol (IP) services, as these services are affected the most by the increased data rates. Naturally, many of the features included in the DVB-S2X standard target these applications and some gains (in terms of spectral efficiency) over DVB-S2 are reported in [3].

The relevant reference markets for DVB-S2X include television broadcasting, interactive services, and professional links [3]. Data content distribution/trunking and other professional applications are mainly point-to-point or point-to-multipoint, including interactive services to professional head-ends, which redistribute services over other media. Such services are characterized by their ability to distinguish receivers based on contents, different quality-of-service targets, and reception capability with consumer-sized antennas. Mobile services and applications have been partly addressed by the DVB-S standard for hand-held terminals (DVB-SH) [4]. Nevertheless, the widespread diffusion of DVB-SH has been significantly delayed by many factors, for example the needed integration between satellite and terrestrial infrastructures [4]. Mobile services remain therefore one of the most appealing emerging markets for satellite operators and service providers. Indeed, broadband data services to land-mobile systems are gathering momentum, as well as broadband and telemetry for aeronautical services.

Since its inception, the DVB-S2X standardization activity has aimed at a performance improvement following either an evolutionary or a revolutionary path. For example, [5] focused on techniques and technologies that may potentially enhance different aspects of satellite communications, namely the achievable spectral efficiency on the satellite channel, as well as the system flexibility and complexity. In particular, the considered technologies include new modulation and coding schemes, new waveforms (e.g., single-carrier orthogonal frequency-division multiplexing, time-and-frequency packing), new algorithms for joint detection, decoding, and equalization, interference mitigation and multiple-input multiple-output (MIMO) systems, framing and pilot optimization. Today, the performance of the state-of-the-art waveforms are very close to the theoretical upper limit [3]. Since a significant performance gain cannot be achieved by means of advanced signal processing techniques [5], the key strategy to improve the satellite services and expand the satellite market is identifying new applications based on existing air interfaces along with additional processing (such as predistortion and channel interleaving) to provide significant gain over the currently deployed waveforms. In this perspective, professional mobile applications are prime targets.

DVB-S2X provides optional functionalities that have not been exploited much so far. Namely, superframing has only been considered to improve synchronization [6] and for interference mitigation purposes [7]. In this paper, we will consider one of the superframe formats described in the DVB-S2X standard [2] and will show how it is instrumental to reliable communications in mobile scenarios. A special attention in the design phase of the system is given to the main impairments affecting satellite mobile communications, namely the nonlinear distortion introduced by the satellite transponder, the correlated fading generated by the propagation channel, the Doppler shift stemming from the mobility of the terminal, and the possibly very low SNR (VL-SNR). To cope with them a predistorter, a plug-and-play self-synchronizing channel interleaver, and a hardwarefriendly yet robust synchronization chain were designed and tested. Finally, in order to assess the suitability of DVB-S2X for realistic mobile applications, a hardware testbed was implemented and 
used to emulate an end-to-end satellite forward link. To the best of the authors' knowledge, this is the first testbed-based study investigating the feasibility of a DVB-S2X-based application in a mobile scenario. Hence, in this paper we will show how the DVB-S2X standard can be a common technology enabler not only for applications based on very small aperture terminals (VSAT), but also for land mobile (LMSS), maritime mobile (MMSS), and aeronautical mobile satellite services (AMSS).

The paper is therefore divided as follows: Section 2 introduces the scenarios of interest, whose channel models are described in Section 3. In Section 4 the technical innovations required to enable reliable communications in the identified scenarios are presented, Section 5 provides a description of the developed hardware demonstrator, and the performance analysis is highlighted in Section 6. Finally, conclusions are drawn in Section 7.

\section{FIXED AND MOBILE SCENARIOS}

DVB-S2X targets the core application areas of DVB-S2 [2], i.e., digital video broadcasting, consumer-oriented interactive services (in the forward link), and professional applications (like IP trunking and backhauling, video contribution such as satellite news gathering, etc.). In this paper, DVB-S2X is used to address new use cases and to provide better services to the mobile segment (in particular, business jets, fast trains, and boats) as well as to the small aperture terminals (e.g., for journalists) which are characterized by very low SNRs. Such capability to work at low SNRs is envisaged to promote new markets for VSAT terminals, whose link budget limits the link availability in the Ka-band and above [8].

Mobile applications are typically based on geostationary (GEO) satellite ground stations with high-gain antennas providing a line-of-sight (LOS) channel with free-space radio propagation. Mobile use cases comprise three scenarios: land, maritime, and aeronautical mobile satellite services. Among these, the most relevant scenarios investigated in this paper are the LMSS and MMSS; AMSS has been identified as one of the possible applications for the DVB-S2X, but apart of the higher Doppler spread and operational SNR, the channel model does not differ much from the reference VSAT scenario, as shown in Section 3.2. Due to all the aforementioned reasons, this paper focuses on professional mobile broadband applications in land mobile and maritime scenarios. As a benchmark, the fixed broadband interactive scenario (VSAT outbound) is considered as well. Each scenario is further detailed in the following.

\subsection{VSAT Outbound}

This scenario deals with broadband interactive services enabled by VSAT configurations and also addresses a core market of DVB-S2. ETSI defines VSAT as a one-way (e.g., a large number of VSATs in US are used for broadcasting) or two-way (interactive) terminal used in a star, mesh, or point-to-point network. While the star network invariably entails a large performance hub station, mesh networks with terminals having similar performance are also present. Figure 1 depicts a typical Inmarsat VSAT network. Particularly, the VSAT terminals SAT1-4 are served by beam A and have varying antenna configurations; SAT5 is served by beam B; further, SAT3 acts as the controller of the network with terrestrial links to all the terminals. Finally, the beams could be in L- and Ku-bands. 


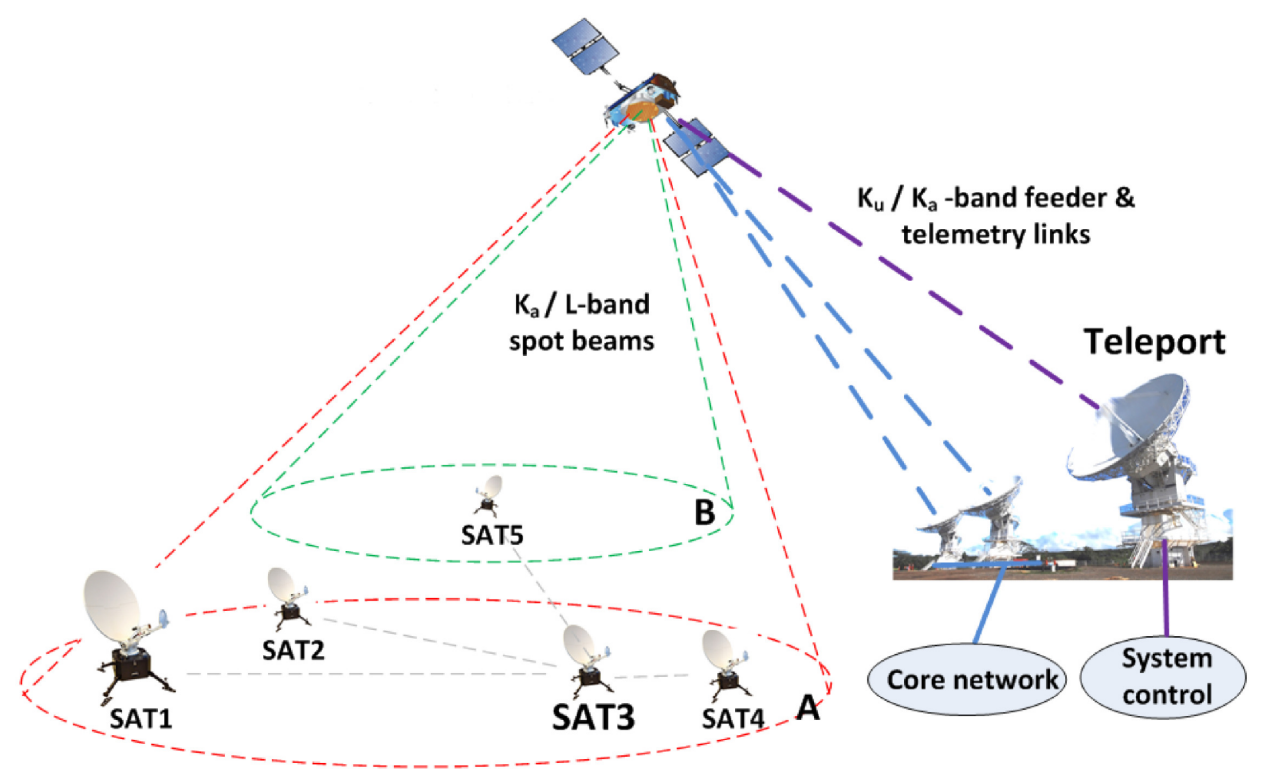

Figure 1. Example of a VSAT outbound application.

A number of satellite service providers offer VSAT networks as cost-effective solutions for connecting reliably a number of terminals worldwide by using a variety of bands (Ka and L, for example). The key services delivered include point-of-sale transactions, as well as bidirectional satellite Internet. The VSAT outbound scenario will be therefore considered as a benchmark, providing fixed services from GEO satellites.

\subsection{Land Mobile Satellite Service}

This scenario presents a typical example of a satellite link deployed in a rural environment where no terrestrial communication is available. The remote user (SAT1 in Figure 2) sets up a satellite connection for data transfer using a portable satellite terminal. The example technologies representing current state-of-the-art are Inmarsat's BGAN, which is based on the constellation of three I-4 GEO satellites providing almost global L-band coverage, and Iridium's Go!, which uses the true global coverage of Iridium's low Earth orbit (LEO) satellite constellation comprising all together 66 satellites operating in the L-band (as shown in Figure 2). The example satellites deploy spot beam technology capable of providing global, regional, local, or customized spot beams. In Figure 2, SAT1 refers to a nomadic user connected to beam B, while SAT2 refers to a mobile terminal that can be served by beams A and B. They could differ in the antenna size and directivity, the former being omni-directional and the latter having some beamshape. The GEO satellites relay the uplink traffic to teleports, which are equipped with large parabolic antennas. These antennas provide very high gain and narrow beams. The teleports are located in central locations typically far away from the users. A LEO satellite system such as Iridium utilizes also inter-satellite links to relay traffic between the user terminals and the teleports. For the sake of clarity, it is worth mentioning that the solution proposed in this paper targets future satellite systems. 


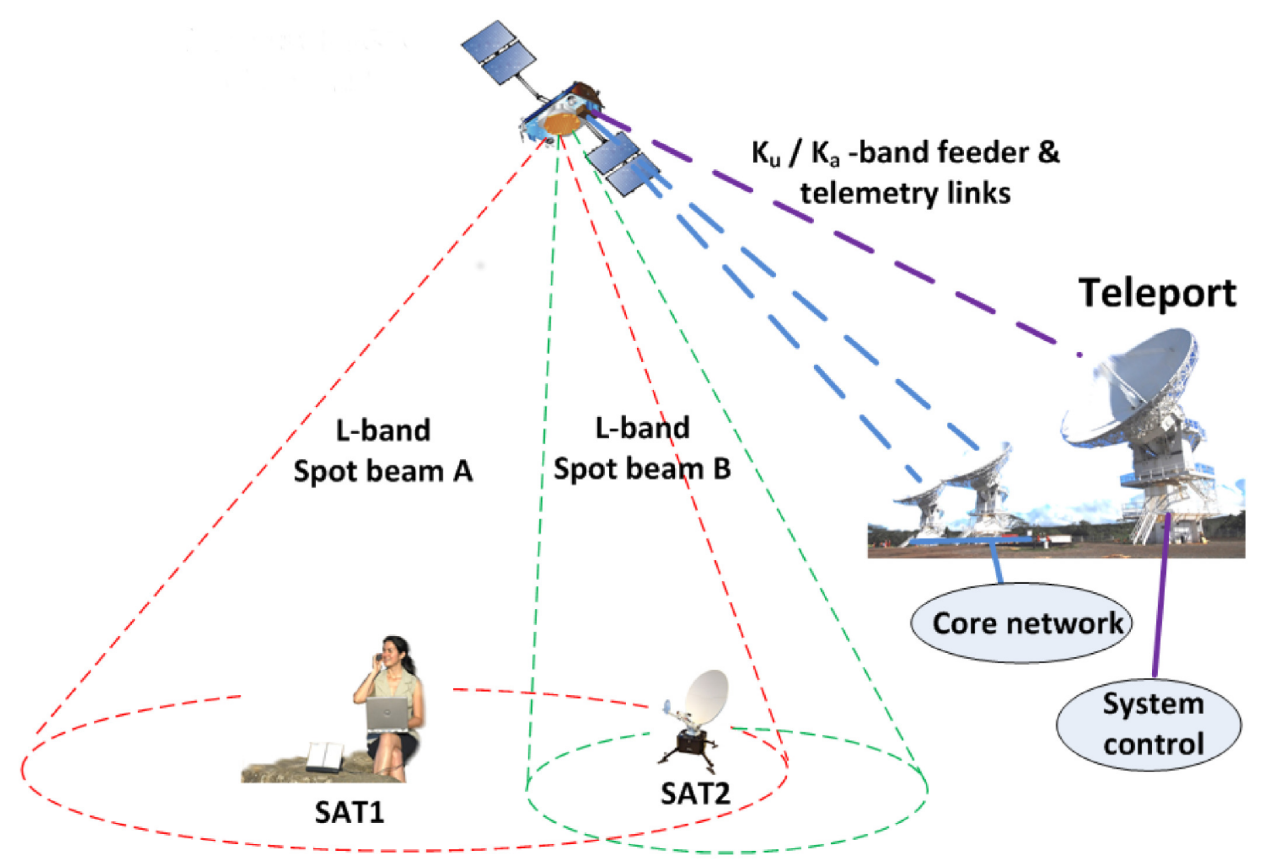

Figure 2. Example of a LMSS application.

The service portfolio includes voice and data connectivity, and Internet access. Typical applications are email and file transfers, video / audio broadcasting (interactive broadband services), conferencing, and low-speed data traffic for safety and critical communications.

\subsection{Maritime Mobile Satellite Service}

Satellite communications provide crucial data and voice communications in maritime applications. As an example, Inmarsat was established to provide communications for cargo ships, oil and gas rigs, and other vessels in service far from the coast line. As ships and vessels are able to carry large antennas and powerful radio transmitters and there are few obstructions blocking the LOS between the ship and the satellite, maritime communications are also an ideal application for satellite communications. For maritime services, high-frequency (HF) radio was the only available communication technology until coastal troposcatter stations were established in the ' $50 \mathrm{~s}$, and still was the only choice for ships in the ocean at that time. HF-CW (Morse code) was officially used in maritime communications until the ' $90 \mathrm{~s}$ and, in addition to current LEO satellite systems (such as Globalstar and Iridium, providing global coverage), it still is the only way to establish communications to ships in the polar areas. Unfortunately, the reliability of HF communications is poor in the polar regions due to the activity of the ionosphere, which is often blocking all $\mathrm{HF}$ communications. Hence, a possible case of interest is the wireless access in the arctic region, which has been provided only by LEO satellites. As the arctic regions are becoming more interesting from the commercial point of view and the amount of traffic is increasing, the utilization of powerful GEO satellites covering areas further towards the polar areas (e.g., the Northern Sea route) becomes also more interesting [9]. In this scenario, high-gain antennas are needed together with waveforms capable of handling very low SNR levels. An example of a satellite system typical to 


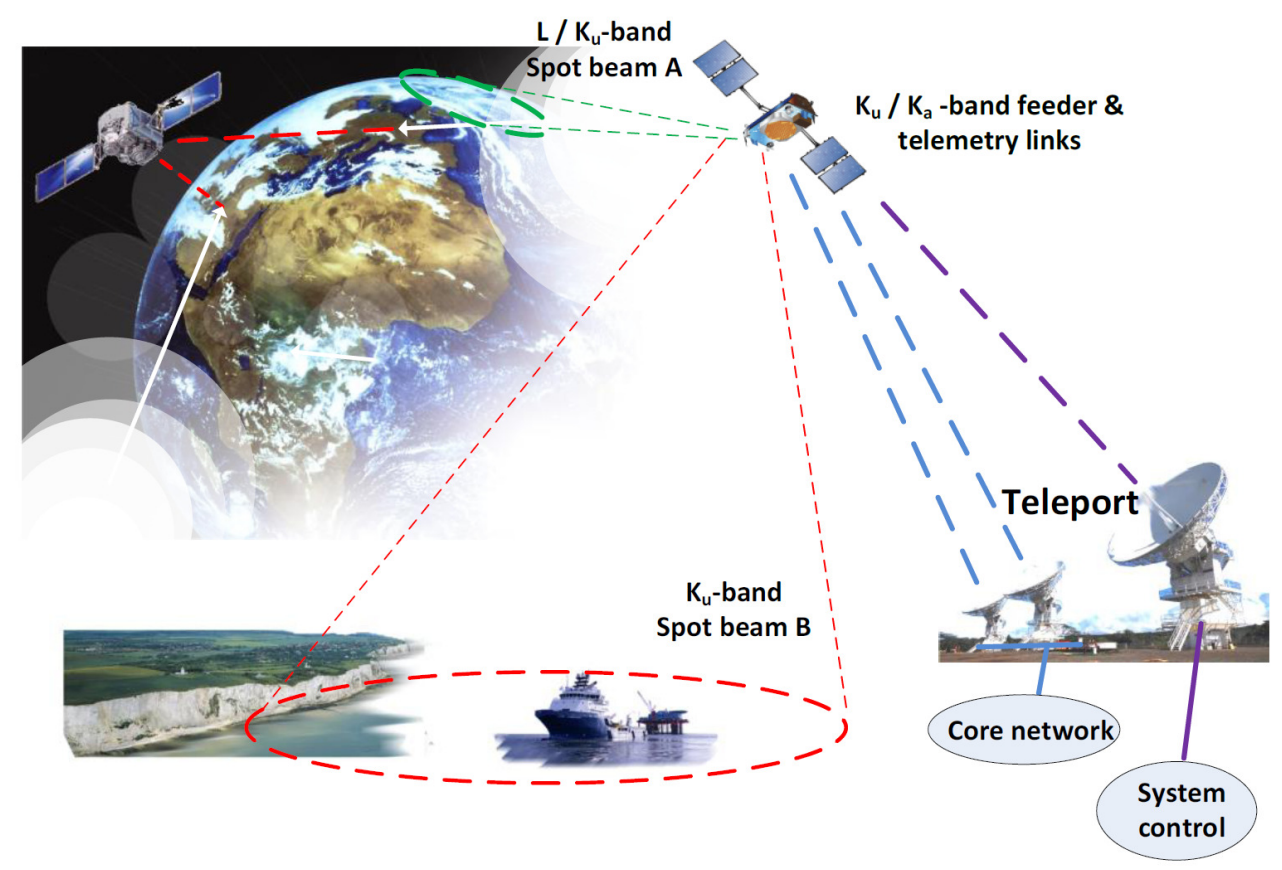

Figure 3. Example of a MMSS application.

this application is Inmarsat's XpressLink, that is a VSAT-type service deploying high-gain dish antennas and operating in the $\mathrm{Ku} / \mathrm{L}$-bands. The Ku-band is deployed for maximum data throughput while a switch over to the L-band can be made to provide the access in the areas where the Ku-band coverage is not provided. An example is depicted in Figure 3.

The key aspects of maritime services include operational, human, and safety communications. Operational communications allow for ship-shore communications, transferring information related to vessel operations, and are seen as essential towards enhancing efficiency. Human communications involve voice, video, and data services, as well as mobile broadband Internet to the crew. Finally, safety communications provide distress services, telemedicine, and other information related to the safety of crew and cargo.

\section{CHANNEL MODELS}

In this section, a technical description of the considered satellite channel models is presented. The adopted models are based on the model used within the DVB-S2X standardization group, which is outlined in [10]. The DVB-S2X channel model includes the significant imperfections and interference in the radio path from an Earth uplink station via a satellite transponder to a user terminal. It is noted that the targets are broadcasting and VSAT applications, which assume LOS type of radio channels. 


\subsection{VSAT Outbound}

The DVB-S2X channel model for interactive broadcasting consists of a transmitter Earth station, a satellite transponder affected by adjacent channel interference and cross-polarization leakage, and a user terminal receiver. The detailed structure of the model is shown in Figure 4, taken from [10]. Differing from this generic broadcasting model, the VSAT outbound model requires only a single transmitter high power amplifier (HPA) with a single carrier as input.

Transponder Model The satellite transponder model consists of an input multiplexer (IMUX) filter, a traveling wave tube amplifier (TWTA), and an output multiplexer (OMUX) filter. In the transponder, the desired carrier is band-pass filtered by the IMUX, and amplified by the TWTA (whose operational working point has to be optimized). The OMUX filter is designed to shape the nonlinearly distorted signal to reduce the interference to adjacent transponders. Typical frequency response models for the IMUX and OMUX filters are available in [10]. The nonlinearity of the TWTA can be approximated by a linearized model or provided as AM/AM and AM/PM characteristics, as shown in [10]. The model for cross-polarization interference (i.e., leakage and coupling) is based on the polarization discrimination of the antenna and depolarization effects in the propagation path (mainly caused by asymmetry in raindrops and ice particles). Recommended values for amplitudes, frequencies, and delays to model cross-polarization and adjacent transponder interference are listed in [10]. The radio propagation channel is assumed to be LOS [10].

Receiver Imperfections Beside the additive white Gaussian noise (AWGN), the terminal receiver imperfections are included in the form of clock, phase, and frequency errors. Also, the amplitude gain distortion occurring in the radio frequency (RF) front-end is taken into account. The mask requirement for the power spectral density of the phase noise is given in [10].

\subsection{Mobile Satellite Services}

The reference model for VSAT applications applies also for modeling mobile satellite services. This is especially true with respect to the hardware non-idealities of transceivers and the satellite transponder. In addition, radio channel propagation characteristics related to the movement of the user terminal need to be taken into account, as shown in Figure 5. In particular, the following aspects have to be considered [10]: (a) Doppler shift, (b) shadowing, (c) multipath propagation, (d) adjacent satellite interference, and (e) SNR dynamics. The characteristics of the satellite radio channel may be grouped in two categories:

- Large-Scale Effects: Due to the fact that LOS conditions are assured in many applications, the satellite propagation channel is dominated by large-scale effects which are related to the long-term behavior of the channel and include: (a) free-space path loss (i.e., attenuation), (b) attenuation due to rain, clouds, and atmospheric gases, (c) shadowing, (d) tropospheric scintillation, (e) propagation delay, and (f) Doppler effect. Large-scale effects are important when the system performance is regarded as a whole, e.g., when evaluating the outage probability. In static LOS scenarios shadowing can be avoided and thus need not to be modeled. Moreover, the occurrence of tropospheric scintillation is so rare that it is not included in the model. In the satellite radio channel characterization, the International 


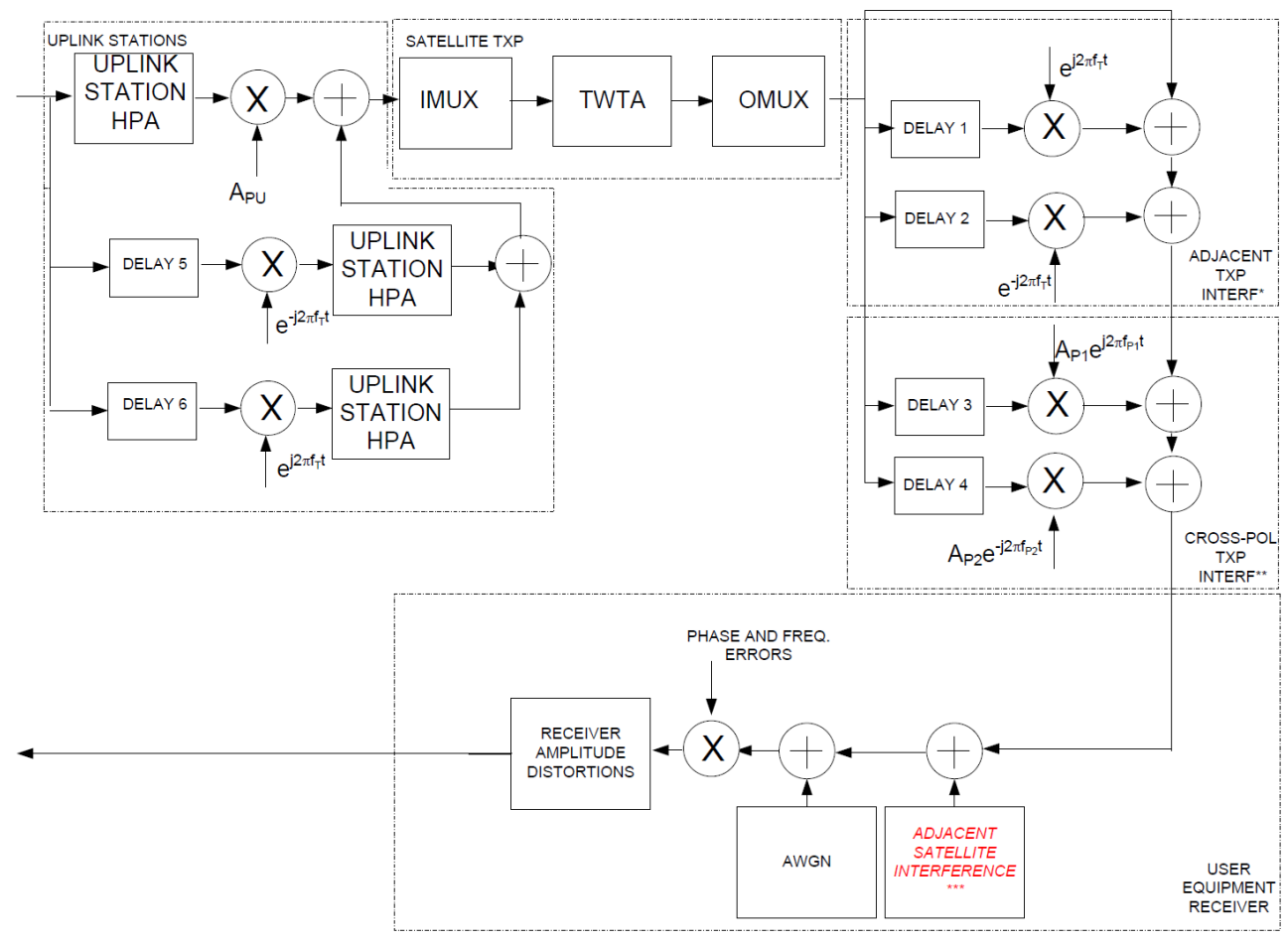

Figure 4. Channel model for interactive broadcasting, taken from [10].

Telecommunication Union (ITU) recommendations can be applied (namely [11]-[14]). In GEO-based fixed satellite service systems like broadcasting, the Doppler effect is not very significant due to the fact that the user terminal (e.g., a home TV) is fixed. In practice, however, the GEO satellite does not orbit ideally but some eccentricity is present in the actual orbit, generating a Doppler shift. The shift is the larger the higher is the carrier frequency. An important cause for significant frequency shift is the frequency translation error in the satellite transponder, as well as the frequency error in the receiver.

- Small-Scale Effects: Multipath fading occurs when several attenuated and delayed replicas of the same signal traveling different propagation paths sum up with random phases at the receive antenna. Depending on the scenario, multipath components may sum up also with the non-faded LOS signal. Tapped-delay line channel models are usually applied when simulating terrestrial links in urban, suburban, and rural environments [15].

The DVB-S2X report makes a conclusion that in the AMSS case LOS propagation can be assumed, so that fading can be neglected [10]. Therefore, it can be modeled in a similar way as the VSAT outbound as long as proper Doppler effects are taken into account. For this reason, no performance analysis (in software and hardware) will be carried out for the AMSS scenario.

In LMSS and MMSS propagation models, both shadowing (slow fading) and multipath (fast fading) have to be modeled. Shadowing is the dominating factor in LMSS due to large buildings and hills, while it may be neglected in MMSS. In this paper, it has been modeled using the landmobile satellite (LMS) channel model based on a Markov state machine [16]. Instead, in MMSS 


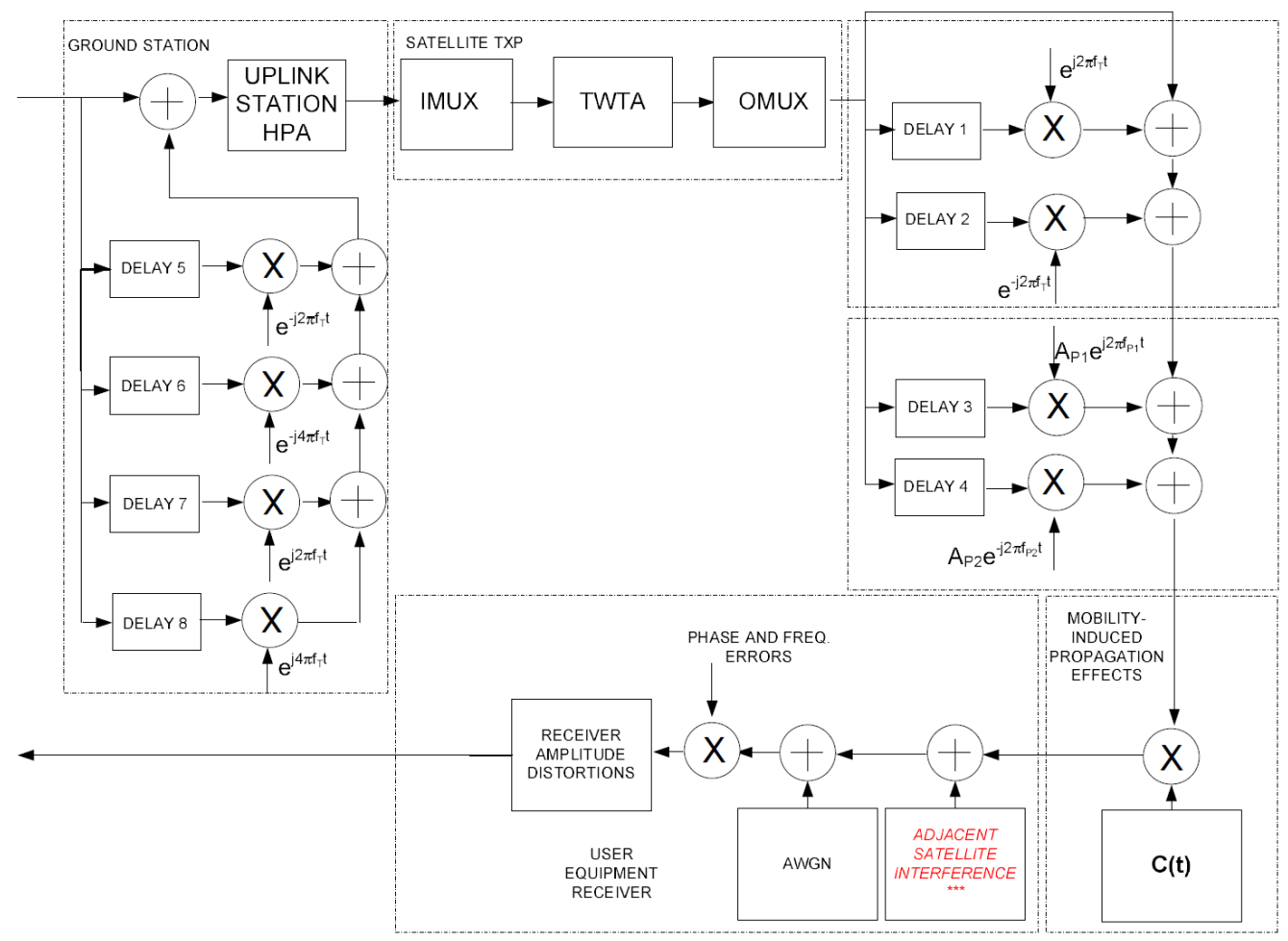

Figure 5. Channel model for mobile scenarios, taken from [10].

the multipath stemming from water reflections has to be modeled ${ }^{\dagger}$, but its impact gets reduced as the directivity of the user terminal antenna increases. Multipath fading seems to be significant in LMSS communications to trains and can be modeled as Ricean fading [10]. In this paper, a user terminal with a highly directive antenna has been considered, allowing to model the multipath fading as a Ricean-distributed single-tap channel. Different values for the $K$-factor of the Ricean distribution have been used for LMSS and MMSS, as reported in Section 6.2. Further, example values for Doppler shifts related to mobility parameters in LMSS and MMSS services can be found in [10].

\section{SYSTEM DESIGN}

In this section, we highlight the main challenges stemming from the considered scenarios and applications, and provide realistic solutions.

\footnotetext{
${ }^{\dagger}$ Multipath is relevant especially at low elevation angles. However, depending on the frequency band and taking into account the ship antenna hight, it can also be seen at higher elevation angles. 


\subsection{Challenges}

The main sources of impairments in the selected scenarios are the transponder, the radio propagation channel, and the user mobility. In the following, we summarize the main impairments affecting the satellite system.

Transponder The transponder introduces linear and nonlinear distortions caused by the IMUX / OMUX filters and the TWTA, respectively, and a frequency offset caused by an imperfect frequency conversion. Traditionally, the linear distortion can be compensated by an adaptive equalizer [17], while the frequency error is corrected by the synchronization chain [18]. The nonlinear distortion requires a dedicated predistortion algorithm. A concise survey of the vast literature on predistortion and the description of the chosen algorithm is given in Section 6.1.

Radio Channel and User Mobility The radio propagation channel and the user mobility generate fading, shadowing, and the Doppler shift. In order to counteract long deep-fade events, a channel interleaver was introduced, whose design is detailed in Section 4.4. The Doppler shift compensation requires a robust synchronization algorithm capable of tracking the frequency variations even in very challenging conditions, such as in the VL-SNR regime. This occurs when the SNR drops below -2 $\mathrm{dB}$ (because of heavy rain events, for example), which is the decoding threshold of the DVB-S2 physical layer (PL) header [19]. In order to guarantee reliable communications at VL-SNR, the DVB-S2X standard has defined the VL-SNR signaling mode [2]. This requires specific MODCODs (using $\pi / 2$-BPSK modulation and coding rates obtained with specific puncturings), a longer PL header, more pilot fields, and a specific scrambling sequence. Two frame structures with different lengths are allowed, as reported in Figure 6. Since VL-SNR signaling occurs when the adaptive coding and modulation (ACM) control loop detects a temporary drop in the SNR on the channel, [10] recommends that the receiver operates in burst mode by decoding frame by frame. This requires a one-shot detection of the frame header and frequency acquisition, which is quite challenging [19]. In order to overcome these synchronization difficulties, the optional superframing structure (briefly summarized in 4.2 and detailed in [2]) can be used [6]. Beside the high number of pilots, the main advantage of superframing is its constant length, which allows the receiver synchronization loops to keep operating in a tracking mode instead of starting acquisition from scratch. In addition, superframing requires that the code shortening and puncturing (which are mandatory for VL-SNR framing) be not applied [2]. This slightly improves the performance of the forward error correction (FEC) codes [2]. Moreover, the extra number of pilot fields improves the training of the equalizer used to compensate for the transponder linear distortion. Also, the fixed duration of the superframe facilitates the design of the channel interleaver, as detailed in Section 4.4.

\subsection{Superframing}

According to its definition in the DVB-S2X standard [2], the insertion of the superframe structure is optional and has the following targets: (a) increasing the resilience to the co-channel interference caused by other beams, (b) supporting synchronization algorithms by regularly inserting pilot fields (which leads to an enhanced receiver performance under severe channel conditions, like VL-SNR regime or link interruptions), (c) future-proof frame design with content format signaling (which 


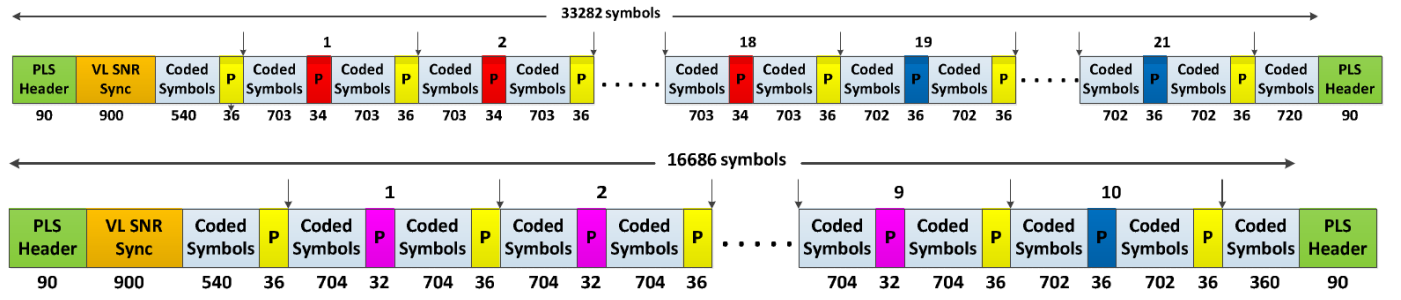

Figure 6. Frame structures in the VL-SNR signaling mode, from [2].

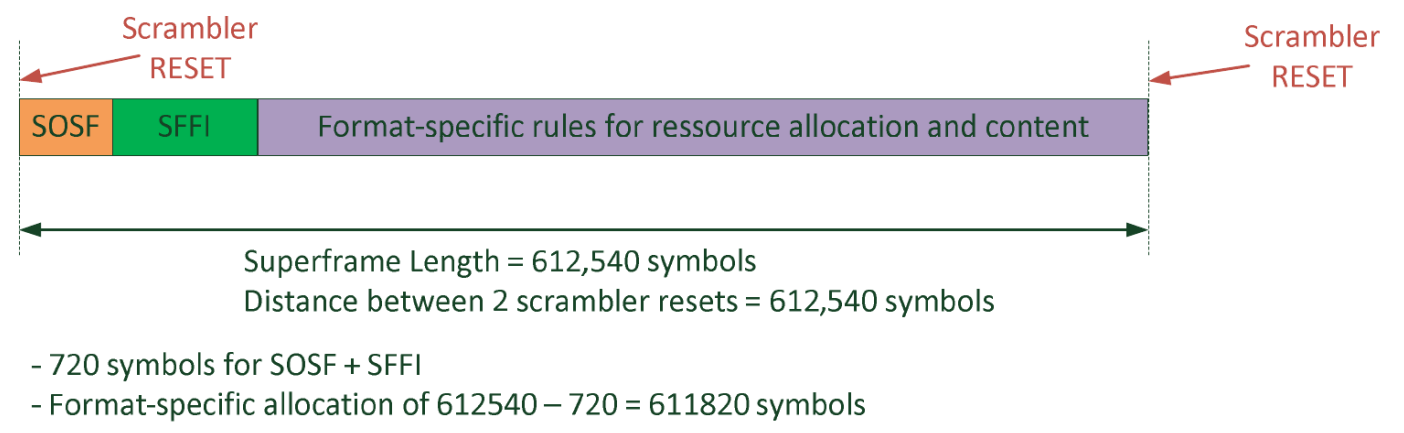

Figure 7. General structure of a superframe, from [2].

is able to accommodate/support interference mitigation techniques, beam hopping operations, and multi-format transmission). The superframing concept is defined to have a constant length equal to 612540 symbols. As indicated in Figure 7, this length holds irrespective of the chosen superframe content format. Each superframe comprises at its beginning a Start-of-Super-Frame preamble (SOSF) and a Super-Frame Format Indicator (SFFI), which fill the first 720 symbols. The remaining part of the superframe is allocated to payload and pilot fields.

According to the standard [2], superframing comes in different flavors depending on the way the superframe itself is built. Since in this paper we target mobile scenarios, possibly at very low SNRs, the number of pilot symbols available in a superframe becomes a key feature for synchronization purposes (both acquisition and tracking); the same seems to be the case even at medium SNRs. In this perspective, superframe format 0 (hosting DVB-S2X physical layer (PL)-frames) and 1 (hosting DVB-S2 PL-frames) can provide a pilot percentage (with respect to the total superframe length) equal to only $2.56 \%$ when the pilot fields are included [2]. Superframe format 4 mostly targets the VL-SNR regimes but provides at most a pilot percentage equal to $2.67 \%$ [2]. On the other hand, superframe format 2 (long bundled PL-frames) and 3 (short bundled PL-frames) provide a pilot percentage equal to 5.03\% [2], making them the best options for the considered applications. From a higher layer perspective, using short FEC codes reduces the system latency and facilitates ACM in mobile scenarios affected by fading. Therefore, particularly interesting for this paper is the superframe format 3, whose composition is shown in Figure 8. It uses short bundled frames (16200 symbols long, instead of 64800 as in format 2) and two types of pilot fields (modulated and nonmodulated). The choice of assessing the performance only for superframe format 3 greatly simplifies the hardware implementation of the testbed. Also, the usage of superframe format 3 in a VL-SNR regime allows neglecting the hardware implementation of VL-SNR header, code shortening, and puncturing. 


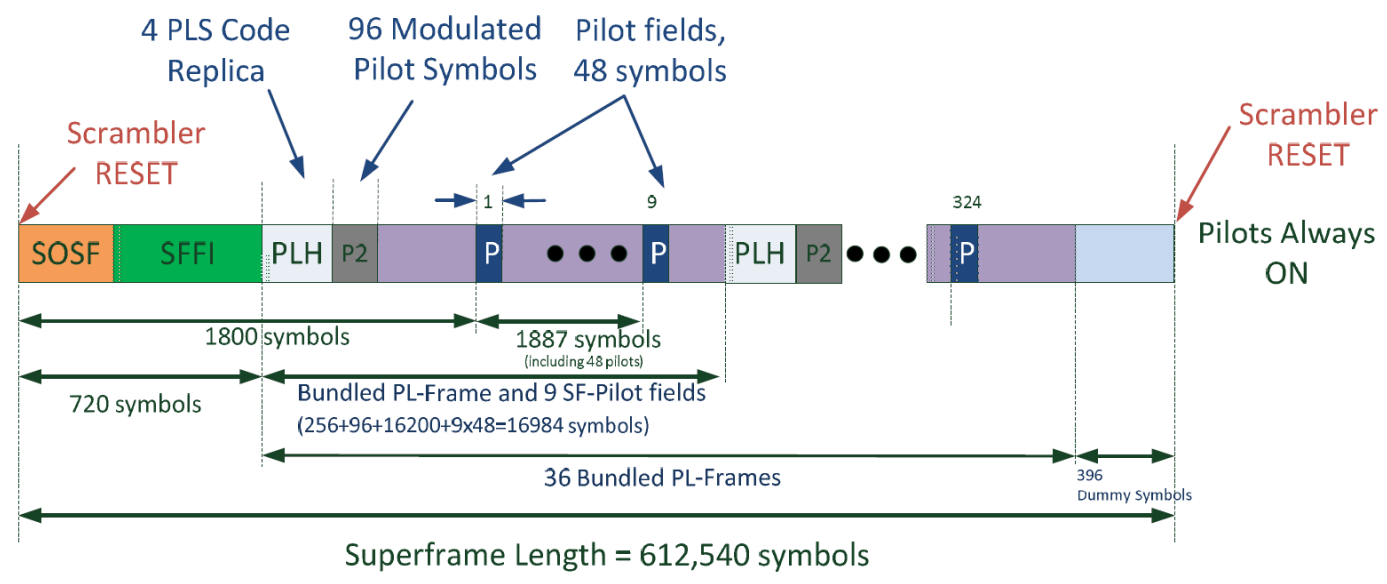

Figure 8. Structure of the superframe format 3, from [2].

\subsection{Predistortion}

Predistortion is widely used for the compensation of the transponder nonlinear distortion [17], [20]. Predistortion can operate on the waveform (as signal predistortion, SPD) or on the data symbols (as data predistortion, DPD). SPD operates on the signal without accessing the original data symbol sequence (i.e., the MODCODs defined by the standard can be used), is performed after the pulse shaping filter, and can be implemented either using nonlinear analog or digital devices. DPD acts on the baseband data sequence and it is always implemented in the digital domain. Different types of predistorters are described in [17]. A DPD based on a look-up table (LUT) has been considered in [21]-[23]. On the other hand, a SPD technique based on the Volterra representation of nonlinear channels with memory is proposed in [24]. A memoryless SPD based on the third-order Volterra series expansion of the nonlinearity is derived in [25], where it is shown to compensate the jitter of the analog-to-digital converter (ADC). In the following, we optimize the uplink signal by resorting to the direct method in [26] using a mathematical procedure for the SPD similar to the one proposed in [25]. In particular, in this paper we consider onground SPD: this means that, while in [25] the SPD is performed inside the satellite payload (namely between IMUX and TWTA), in this paper it is performed at the transmitter, right after the pulse shaping and before the satellite payload. The chosen formulation for the onground SPD has two significant advantages: the symbol constellation does not need to be changed, and only two complex coefficients are required to predistort the signal.

In the following, we provide the models used to derive the predistorter, the procedure followed for the parameter optimization, and suggest a low-complexity implementation.

Modeling The input-output characteristics of the transponder is a nonlinear non-invertible function with memory, where the nonlinearity comes from the AM/AM and AM/PM characteristics of the TWTA, while the memory comes from the impulse responses of IMUX and OMUX. In order to make the compensation problem tractable, we neglect at this stage the memory effects introduced by the transponder filters, and assume that they will be compensated separately at the receiver side. To this purpose, an adaptive equalizer is used as shown in Figure 10. By neglecting the memory effects, we can approximate the input-output characteristics of the transponder with a 
memoryless third-order Volterra series expansion as

$$
y(t, \alpha, \beta)=\alpha x(t)+\beta|x(t)|^{2} x(t)
$$

where $x(t)$ is the transponder input signal, $y(t, \alpha, \beta)$ is the approximated output, and the coefficients $\alpha$ and $\beta$ are assumed to be complex. As SPD model we choose a matching memoryless third-order polynomial function, namely

$$
x(t, \gamma, \delta)=\gamma s(t)+\delta|s(t)|^{2} s(t)
$$

where $s(t)$ is the input signal (i.e., the output of the shaping filter), and $\gamma$ and $\delta$ are the complex predistorter coefficients to be computed. The chosen model is known for providing for a satisfactory trade-off between performance and complexity [27].

Optimization and Implementation Denoting with $\tilde{y}(t)$ the actual output of the transponder, the optimal Volterra series coefficients $\alpha$ and $\beta$ can be jointly estimated by minimizing

$$
\alpha, \beta=\underset{\mathbb{C}^{2}}{\operatorname{argmin}} E_{x}\left\{\|\tilde{y}(t)-y(t, \alpha, \beta)\|^{2}\right\}
$$

where $E_{x}\{$.$\} denotes the expectation with respect to x(t)$. This is a classical least squares minimization problem where the error function is linear in the coefficients $\alpha$ and $\beta$. The SPD parameters, $\gamma$ and $\delta$, can be found by solving a minimization problem similar to (3), i.e.,

$$
\gamma, \delta=\underset{\mathbb{C}^{2}}{\operatorname{argmin}} E\left\{\|y(t, \alpha, \beta)-s(t)\|^{2}\right\}
$$

where $E\{$.$\} denotes the expectation with respect to the signal s(t)$, and $y(t, \alpha, \beta)$ uses as transponder input the predistorted signal $x(t, \gamma, \delta)$ in (2). It is worth noting that, since the optimization is carried out offline, no feedback is required from the user terminal and no noise term appears in (4). The resulting error function is nonlinear in the coefficients $\gamma$ and $\delta$ because of (1), and the method used to estimate the Volterra coefficients for the transponder cannot be used any longer. Therefore, the minimization has to be done numerically. Unfortunately, finding a solution for (4) is a formidable task. Hence, we transform the minimization problem in (4) into an equivalent constraint-satisfaction problem that can be solved by exploiting the Divide \& Concur (D\&C) algorithm [28]. The latter is an iterative graph-based algorithm which has given good results in many different applications [28]. Details on the constraint-satisfaction reformulation of the optimization problem in (4) can be found in [25] and directly apply to our problem. The partial solutions computed by the D\&C are evaluated by using the Constrained Optimization BY Linear Approximations (COBYLA) algorithm [29], [30]. Since D\&C provides no guarantee to find a global minimum, the multi-start approach is adopted. Therefore, the optimization procedure is repeated multiple times, and eventually only the best result is chosen. Clearly, the computation of the predistortion coefficients can be done only offline. A hardware-friendly implementation of the SPD relies on a LUT tabulating the predistortion function (2) with the optimized coefficients. 
Table I. Parameters for the channel interleaver.

\begin{tabular}{|c|c|c|}
\hline Modulation & $M$ & $L$ \\
\hline BPSK & 72 & 135 \\
QPSK & 144 & 135 \\
8PSK & 216 & 135 \\
16APSK & 288 & 135 \\
32APSK & 360 & 135 \\
\hline
\end{tabular}

\subsection{Channel Interleaver}

The channel interleaver is a convolutional interleaver operating over multiple superframes in order to break the time correlation of the fading realizations affecting the radio channel [4]. In order to synchronize interleaver and deinterleaver, a dedicated signaling is required. Alternatively, the signaling can be avoided if the interleaver is properly dimensioned; in this perspective, the constant length of the superframe becomes a key feature.

Since the focus of this paper is on superframe format 3, the only modulation formats taken into account for the interleaver design are BPSK, QPSK, 8PSK, 16APSK, and 32APSK [2]. However, the design can be extended to the other superframe formats. The fundamental unit of the channel interleaver is the interleaver unit (IU), which contains $N_{b}$ bits. The channel interleaver is formed by $L$ branches of increasing length, and the $i$-th branch contains $i M$ IUs where $M$ is an integer constant. The self-synchronizing design of the interleaver can be achieved by ensuring that the first $N_{b}$ bits of the superframe are contained in the IU on the first branch of the interleaver. This design is motivated by the one provided in the DVB-SH standard [4].

In the following we choose $N_{b}=60$ bits (which corresponds roughly to 8 bytes) in order to simplify the hardware implementation. This choice ensures an integer number of symbols per IU for all the modulation formats considered in the superframe format 3 (i.e., 60 is a common multiple of 2, 3, 4, and 5, corresponding to QPSK, 8PSK, 16APSK, and 32APSK, respectively). The $M$ and $L$ parameters can be defined according to the modulation format. In order to have a simpler implementation, we set $L=135$ while $M$ is left free to vary according to the cardinality of the modulation, as shown in Table I. The structure of the interleaver is shown in Figure 9.

\subsection{Synchronization Chain}

A block scheme of the considered synchronization chain is shown in Figure 10. The main features include:

- frequency error compensation, including tracking of the Doppler shift caused by the mobile terminal;

- timing recovery, to cope with the group delay introduced by IMUX and OMUX, as well as the timing drift caused by hardware imperfections at the receiver;

- detection of the start of superframe;

- phase compensation, including tracking of the phase noise;

- equalization, to compensate for the linear distortion of the transponder. 


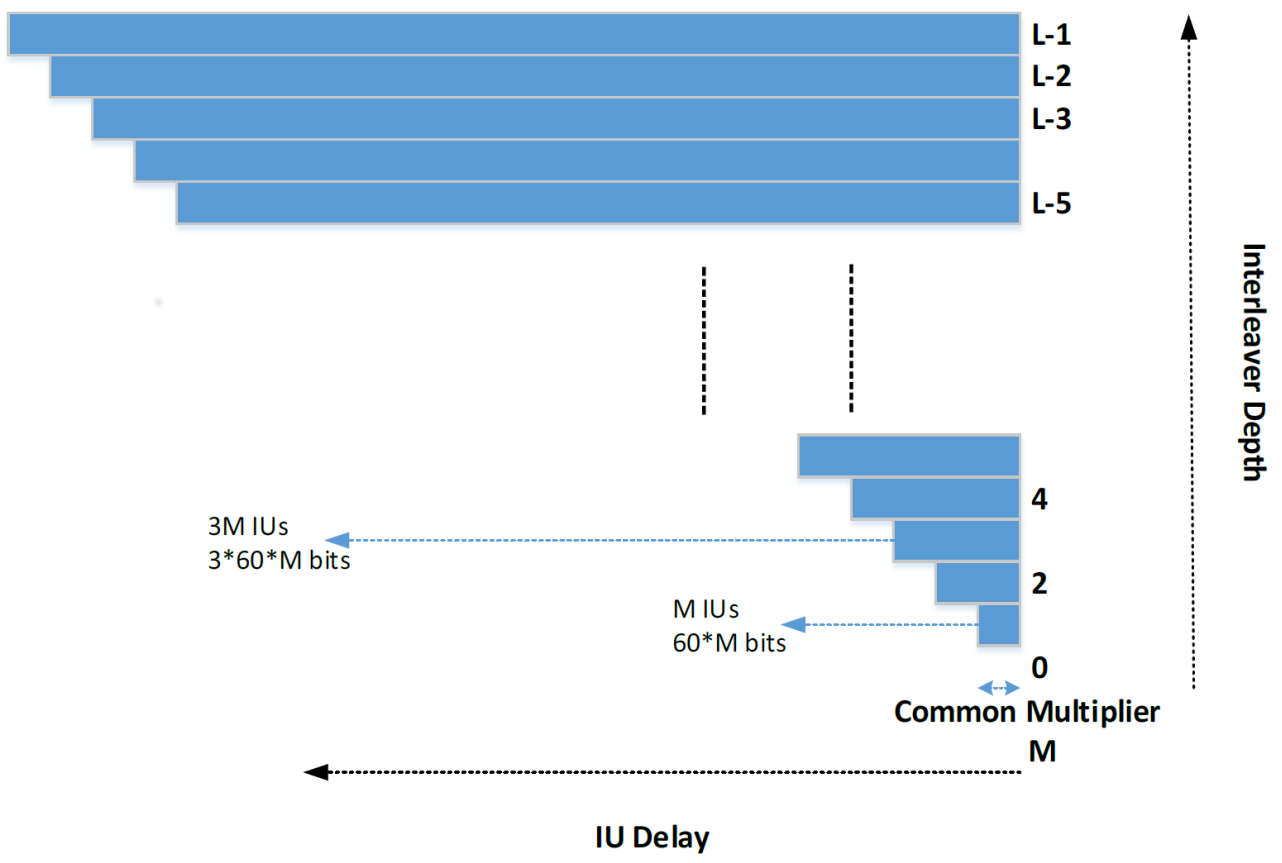

Figure 9. Structure of the channel interleaver.

In the chosen scenarios, the main challenge for the receiver is the synchronization in the VLSNR regime and/or in a mobile environment. To this purpose, a strong blind coarse frequency estimator able to track and compensate for a possibly high frequency error (even at VL-SNR) is mandatory. The chosen algorithm is the balanced quadricorrelator [31] working in the oversampled domain, since it is able to satisfy all these stringent requirements. The second block of the chain performs timing synchronization by resorting to the Gardner algorithm [18]. This block works in the oversampled domain (with two samples per symbol) and compensates for the fractional timing offset, the timing drift caused by the hardware imperfections, and the channel group delay.

All the blocks subsequent to timing synchronization implement pilot-aided algorithms to estimate the start of the superframe, the phase, and to refine the frequency estimate. Therefore, the received signal is downsampled and one sample per symbol is processed. After timing synchronization the chain implements the frame synchronization by resorting to the non-coherent post-detection integration (NC-PDI) algorithm [32], [33]. This algorithm is based on the correlation between the received symbols and the known transmitted symbols (i.e., SOSF and pilots). The NC-PDI compensates for the integer timing offset (with respect to the symbol period) and detects the start of the superframe. In order to limit the hardware complexity, the correlation is computed only over a subset of the available known symbols in a superframe (i.e., over 720 symbols contained in SOSF and in the first pilot fields). Then, pilot-aided fine frequency and phase synchronizations exploit the Mengali \& Morelli algorithm [34] and linear interpolation, respectively.

At the end of the synchronization chain, a symbol-spaced minimum mean squared error linear equalizer is used to compensate for the linear distortion introduced by the satellite filters and the radio propagation channel. The chosen update rule is the classical recursive least squares because of its fast convergence. Finally, the SNR estimation required for the soft demapping is carried out by the SNR estimator (SNORE) [35]. 

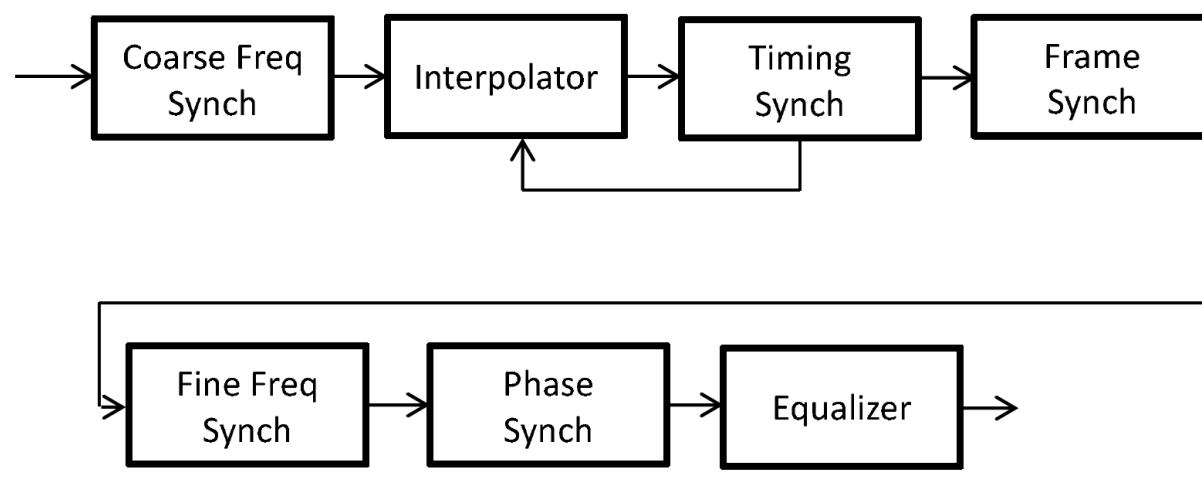

Figure 10. Block scheme of the synchronization chain.

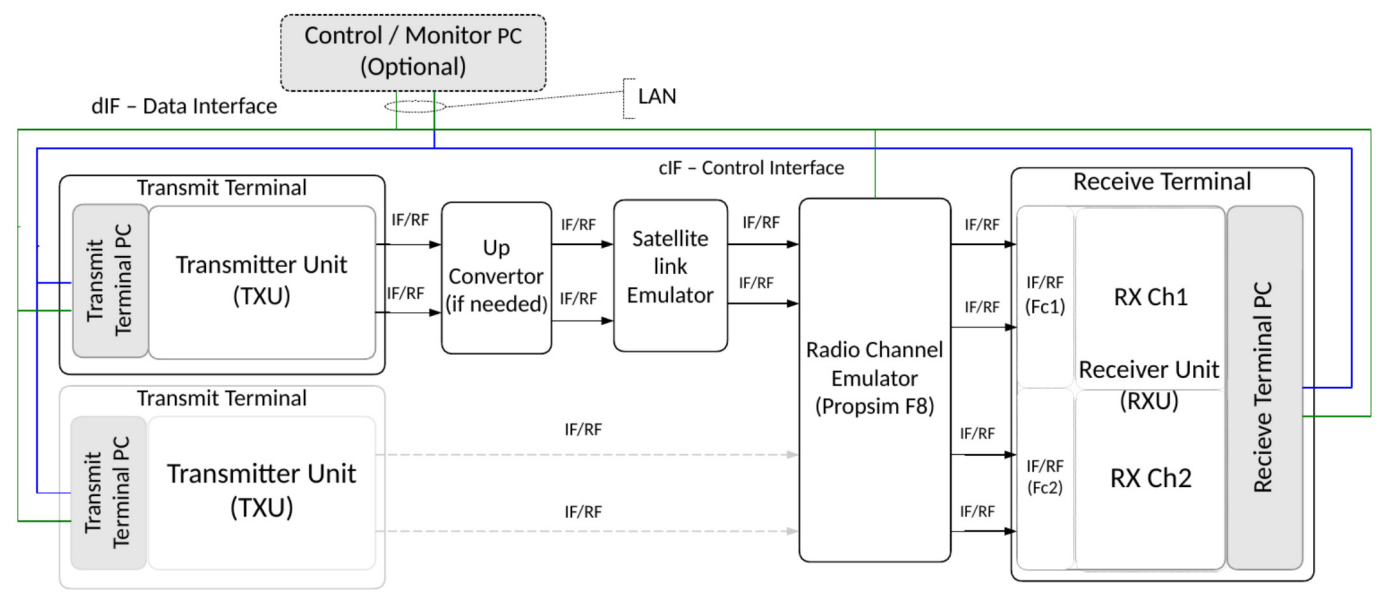

Figure 11. Block scheme of the testbed.

In the synchronization chain described above (which is the one used for the software simulations), the constant length of the superframing structure is not exploited. However, the developed hardware testbed uses the constant length of the superframe to improve its frame synchronization and to reduce the implementation complexity by shortening the search time interval for the start of superframe. All the parameters characterizing the synchronization chain were tuned for each scenario, and the corresponding values are reported in Section 6.2.

\section{HARDWARE DEMONSTRATOR}

The hardware demonstrator includes transmit terminals, a receive terminal, satellite emulator, a radio channel emulator (Propsim), and a control personal computer (PC). Transmit and receive terminals are frequency-locked by exploiting a global positioning system (GPS) signal. Figure 11 shows the system's block scheme, while Figure 12 provides a picture of the assembled testbed. 


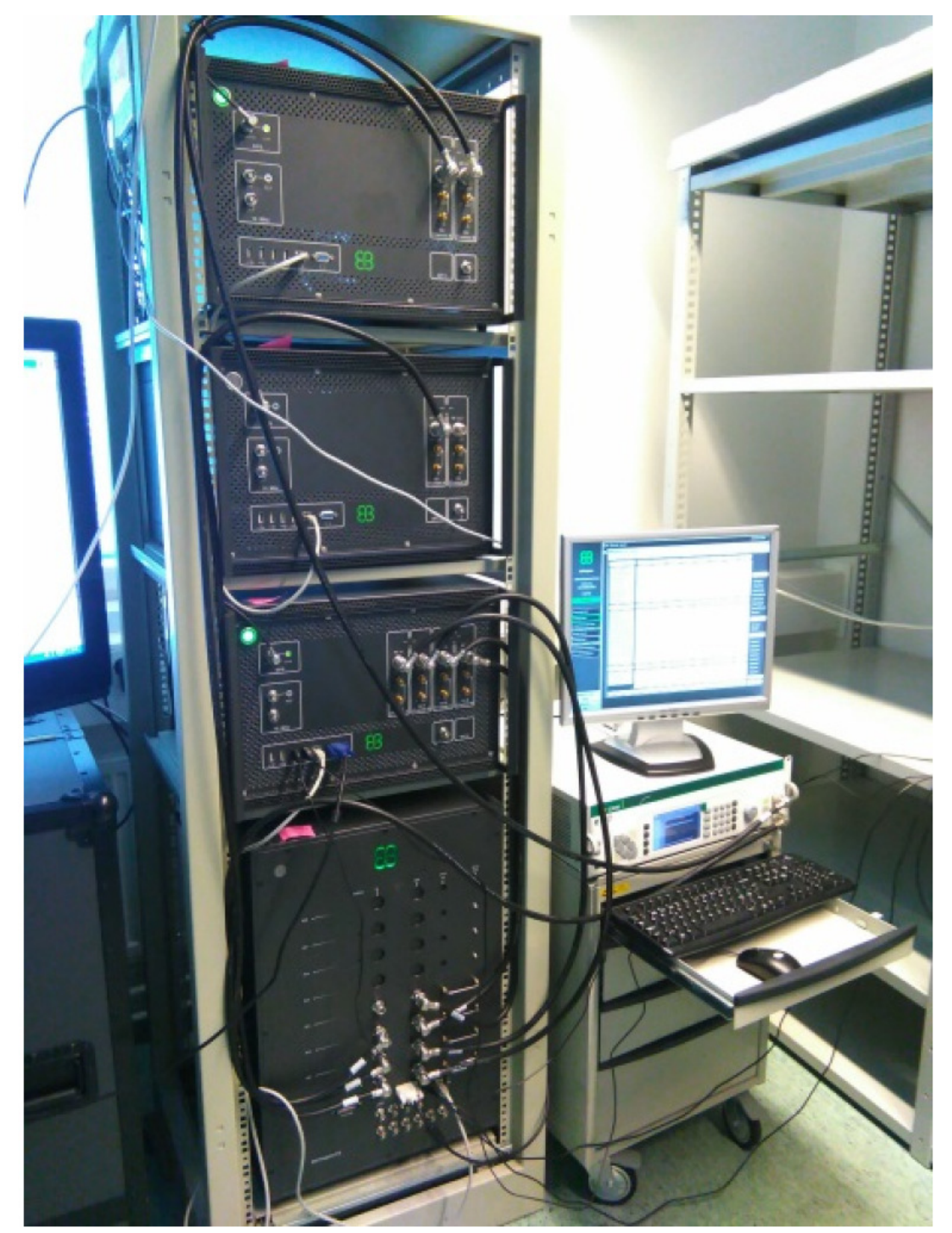

Figure 12. Picture of the testbed.

\subsection{Testbed Architecture}

Both transmit and receive terminals are implemented on common software-defined radio (SDR) platform. Each terminal emulator is composed of four sub-blocks, detailed in the following.

5.1.1. Transmitter Architecture The top-level schematic of the terminal hardware is shown in Figure 13. The hardware is broken down into four main sub-systems:

- power supply module (PWR);

- clock generation module (CGM);

- transmit terminal PC module (TTPC);

- transmitter module (TXU).

The PWR module provides the necessary DC power to the system. Connected to AC mains, the PWR module generates and regulates multiple DC voltage rails. 


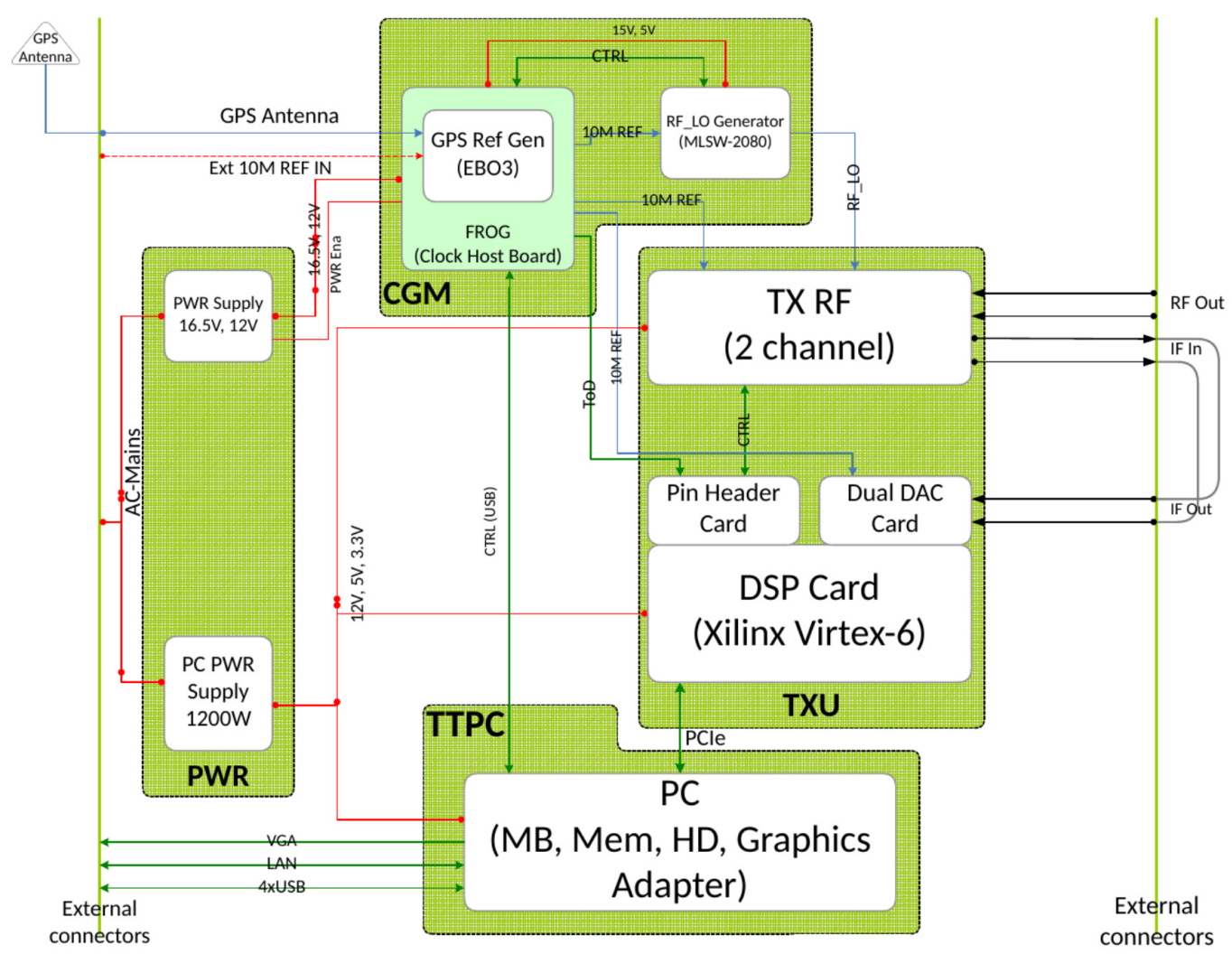

Figure 13. Block diagram of the transmitter module.

The CGM module has three main functions:

- To generate and distribute a $10 \mathrm{MHz}$ reference clock (shown in Figure 13 as 10M REF) locked to either an external $10 \mathrm{MHz}$ reference or a GPS clock signal. A common $10 \mathrm{MHz}$ reference is then used by all other clock-generation circuitry to maintain frequency synchronization within the terminal.

- To generate the sine wave of the RF local oscillator, shown in Figure 13 as RF_LO.

- To broadcast a time-of-day timestamp to the baseband module for triggering purposes. The time-of-day information (shown in Figure 13 as ToD) is generated in the GPS clock module and contains a 1 pps (pulse per second) signal as well as the timestamp in a serial interface.

The TTPC module contains the control PC for the transmit terminal. The TTPC hosts the server software that provides an interface between the signal-processing hardware and the outside world.

The TXU module is the heart of the SDR transceiver. The TXU contains the hardware for the required RF up-conversion and baseband processing necessary to implement the DVB-S2X transmitter. Output signals at intermediate frequency (IF) are looped through external connectors to allow using the external RF up-conversion for the IF source. The TXU and the embedded firmware modulate the DVB-S2X transport data stream onto one or two transmit antennas. 


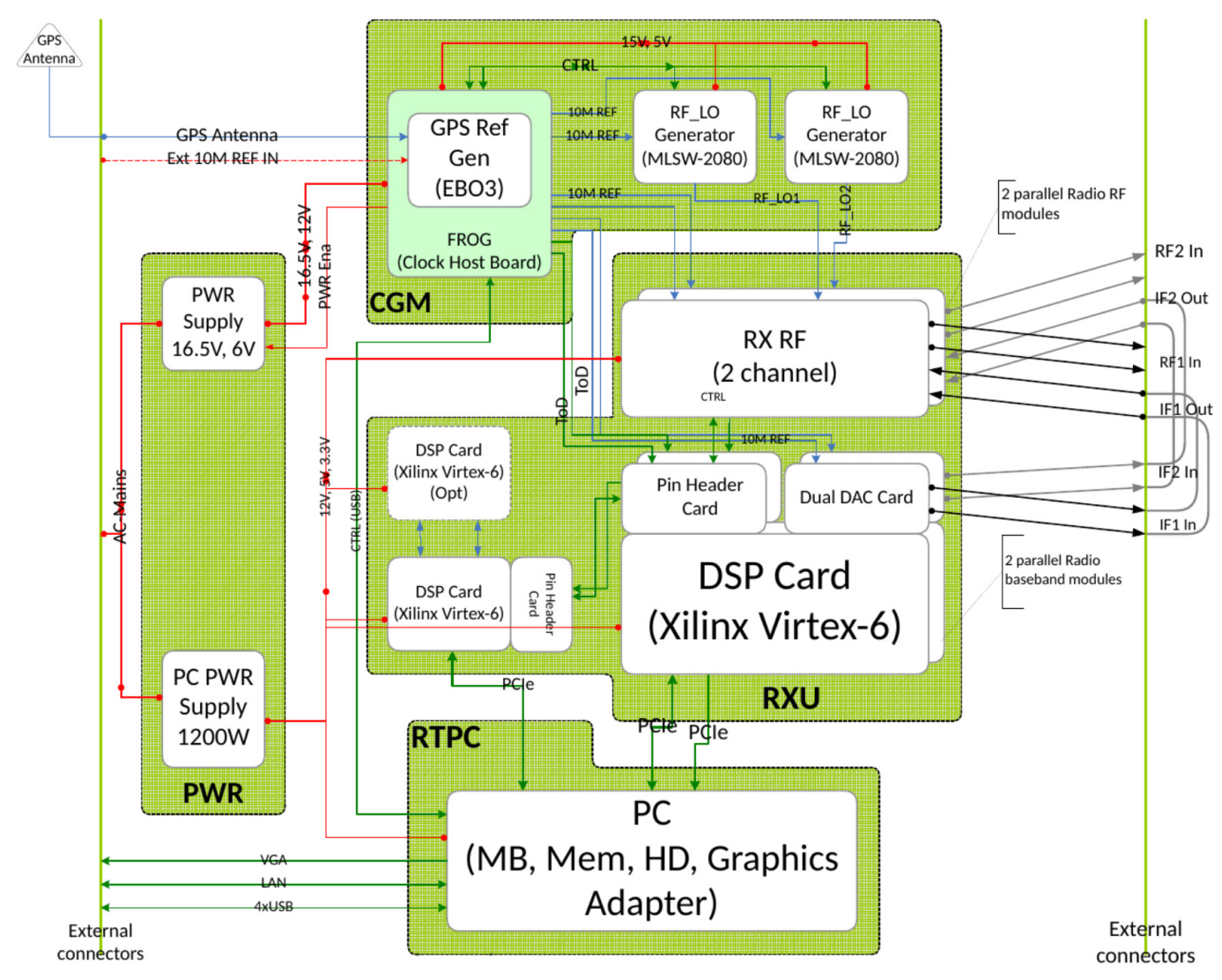

Figure 14. Block diagram of the receiver module.

5.1.2. Receiver Architecture The RX module has $2 \times 2$ input channels for MIMO purposes. In this implementation, only one input channel is used. Each module includes a PC motherboard, which enables using display, mouse, and keyboard to control each module individually. For debug purposes, the Xilinx ChipScope debugging tool is available in all modules. The top-level schematic of the receive hardware is shown in Figure 14. As for the transmitter, the hardware is broken down into four main sub-systems: PWR, CGM, receiver terminal PC module (RTPC), and the receiver module (RXU).

The PWR and RTPC modules provide the same functions as their equivalent sub-systems at the transmitter. The CGM module is similar to its transmitter counterpart, the only difference being that it generates two local oscillator sine waves (shown in Figure 14 as RF_LO1 and RF_LO2) to support multi-frequency network (MFN) operation.

The RXU contains the hardware for the required RF down-conversion and the baseband processing necessary to implement the DVB-S2X receiver. In order to support MFN operation, the RXU contains two dual-channel RF down-conversion sub-modules. These sub-modules can be tuned to independent RF carrier frequencies (shown in Figure 14 as RF_LO1 and RF_LO2). The RF sub-modules provide an IF output signal to two independent receiver modules performing the baseband signal processing. IF output signals are looped through external connectors to allow using external IF source with external RF down-conversion. A third DSP card contains the hybrid combining and error correction signal processing. 
5.1.3. Satellite and Radio Channel Emulators The satellite channel emulator is an IZT C3040, used to model impairments stemming from the satellite hardware. The radio channel emulator is a fourchannel Propsim emulator that can provide an independent radio channel between each input and output port. Interference can be added to each output port. Both channel emulators are controlled by an Ethernet connection.

\subsection{Firmware Design}

The field programmable gate array (FPGA) design firmware implements the actual physical layer waveform of DVB-S2X. The firmware is run in digital signal processing (DSP) units at both transmitter and receiver modules. Each firmware includes a wrapper logic to interface with the other blocks in the testbed. The wrapper logic is provided by the EB-RACE platform and includes all the interfaces required between the FPGA and the external logic. The FPGA top-level is illustrated in Figure 15. Each of the FPGAs in the demonstrator shows the same general architecture. The toplevel scheme contains a number of interface blocks surrounding the actual signal-processing engine.

The FPGA is connected with the PC environment through the peripheral component interconnect express (PCIe) bus. The PCIe is a first generation $\times 8$ interface allowing transfer speeds up to 2 GBps in both directions. The PCIe endpoint block is connected to the DDR3 SDRAM interface block, allowing rapid access to the off-chip 512 MB SDRAM. The DDR3 SDRAM arbiter also allows access to the SDRAM from within the FPGA fabric. This allows the signal-processing engine to utilize the off-chip SDRAM. A register interface block forms a bridge between the PCIe endpoint and the FPGA internal register space. The software access to FPGA registers is done via the PCIe bus with the register interface block. The register interface internal bus is global and drawn in Figure 15 as a green ring. A reset interface block synchronizes a single software-controlled reset to each of the clock domains present in the design. These resets are global and drawn in Figure 15 as a red ring. Synchronizing the reset to the proper clock domain eliminates possible metastability issues with asynchronous resets. The clock interface block manages the on-chip global clocks. The clocks are sourced off-chip from oscillators and external clock generators. The clock interface monitors the input clocks, generates internal clocks, and distributes them in a global manner. The global clocks are drawn as a blue ring in Figure 15. The environment logic that is common to all FPGAs in the design requires roughly $7 \%$ of the slice and $14 \%$ of the block RAM and $1.4 \%$ of the DSP48 resources.

5.2.1. Transmitter Firmware The transmitter firmware has been implemented as VHDL code in the Virtex-6 LV240 FPGA circuit of the transmitter module. The transmitter FPGA contains the blocks presented in Figure 16. In addition to the blocks described in the figure, a convolutional interleaver block has been drafted to support future expansions of the design.

The transmitter blocks follow the specifications presented in the DVB-S2X standard [2], and supports the superframe format 3 . The actual symbol rate in the demonstrator is always $8 \mathrm{Mbaud}$, but different symbol rates can be emulated by scaling transceiver and channel impairments accordingly. Parameters such as MODCOD and roll-off can be changed run-time via the register interface. The MODCOD value can be changed automatically via the ACM-loop script that runs in the control software, or it can be set to a constant value during the measurement. The transmitter architecture supports the use of two input data flows that can be controlled individually, i.e., MODCODs and user 


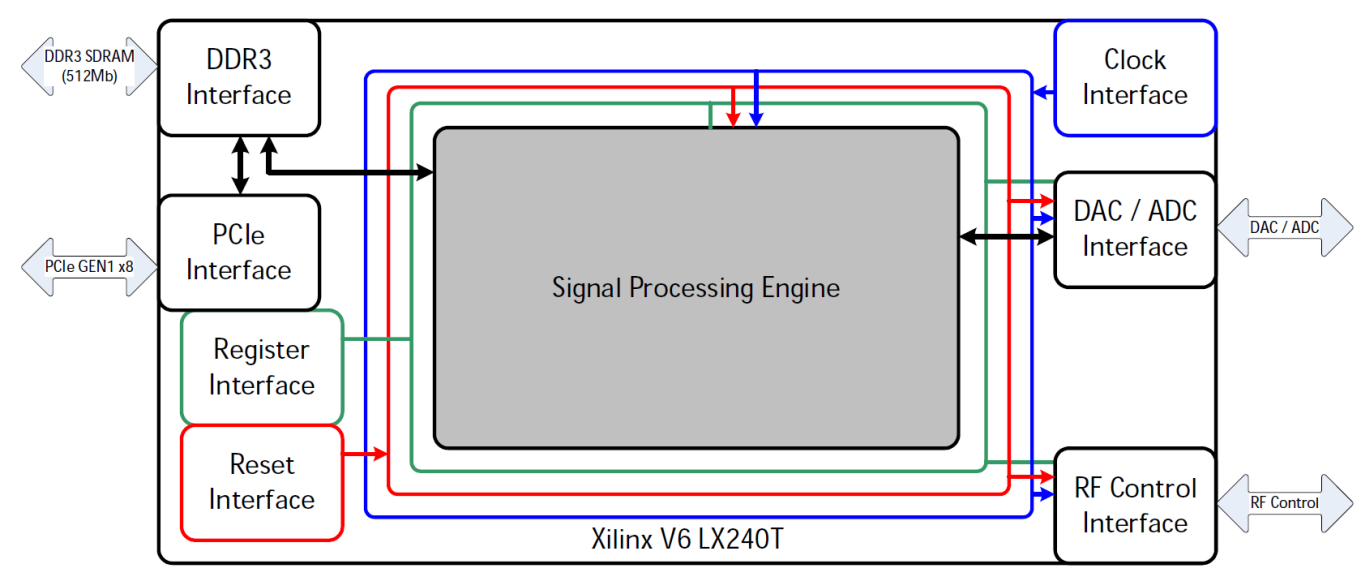

Figure 15. FPGA wrapper logic.

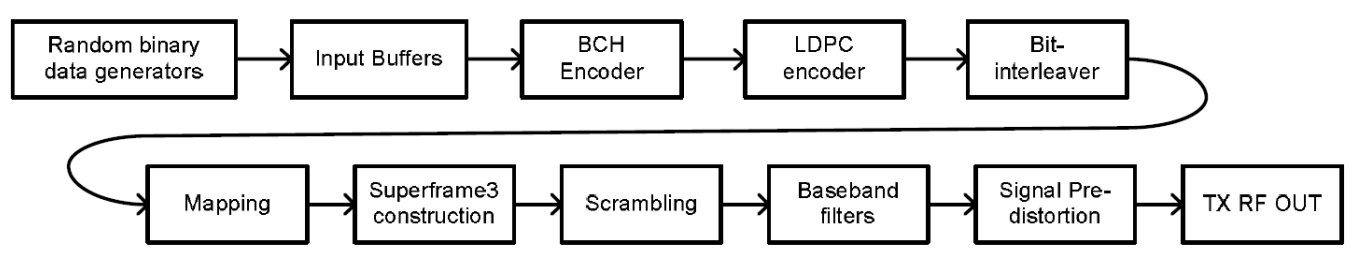

Figure 16. Transmitter block diagram.

data rates for both flows can be set separately. The output of the transmitter dataflow is a continuous 8 Mbaud signal with a bandwidth equal to $8 \mathrm{MHz}$. The baseband filtering block also handles the oversampling of the signal to the platform digital-to-analog conversion (DAC) unit, which further forwards the data to the transmit RF parts.

5.2.2. Receiver Firmware The receiver synchronizes the carrier frequency, symbol timing, and detects the start of the superframe. Bundled frames carried by the superframe are decoded one by one, so they can contain any supported MODCOD in any order. Frame error rate (FER) measurements are performed for a preset MODCOD. The FER check block also measures the data rate for the chosen MODCOD as bytes per second. The receiver is implemented on two FPGA circuits Xilinx Virtex-6 LX240T. High-level receiver blocks are illustrated in Figure 17. The SDR platform has resources for $2 \times 2$ MIMO channel, but for this application only one data stream is implemented. The RF tuner is tuned to the center frequency and the signal is digitized by an ADC converter.

FPGA 1 (PHY) contains the physical layer processing required to synchronize and demodulate the received signal, and compute the bit log-likelihood ratios (LLRs). It includes:

- I/ Q-down conversion;

- matched filtering;

- synchronization;

- demodulation;

- deframing;

- channel estimation; 


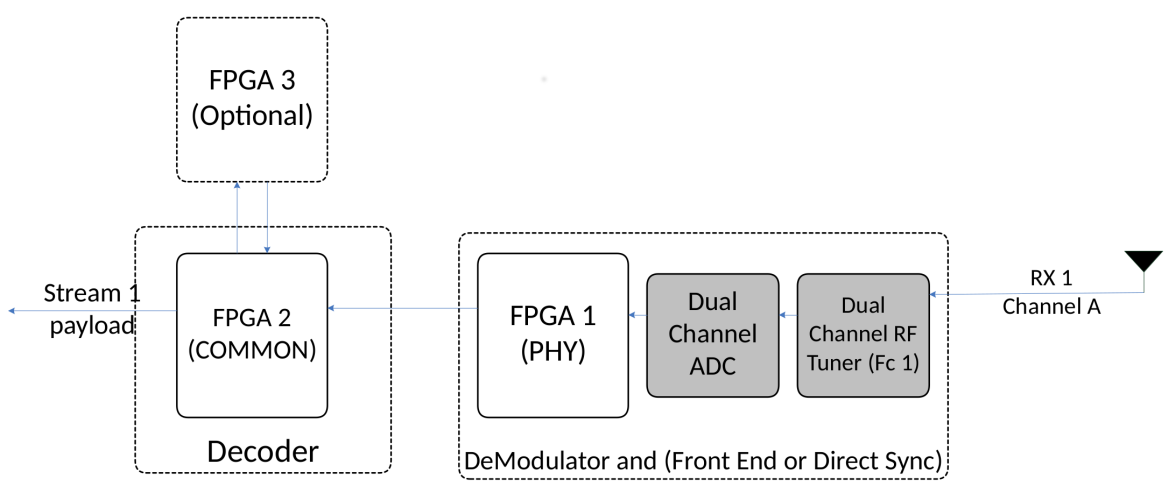

Figure 17. Receiver block diagram.

- demapping.

The input to FPGA 1 is a stream of ADC-samples in IF, and its output is a stream of LLRs. The stream is routed to FPGA 2 where the COMMON processing is performed. The COMMON processing deinterleaves and decodes the LLR streams. The decoded FEC words are checked for errors and error rate statistics are forwarded to the control PC through the trace functions. Therefore, FPGA 2 (COMMON) contains:

- deinterleaving;

- FEC decoding (licensed by Fraunhofer IIS);

- bit and frame error rate counters.

A third optional FPGA 3 can be inserted and connected to FPGA 2 to offload some of the processing, if required. According to the current FPGA resource budget, FPGA 3 is not needed. Regardless, this provides an easy expansion possibility if need be.

Each FPGA is connected to the serving PC via the PCIe interface. This connection, in addition to the off-chip SDRAM, allows data tracing from various points along the chain. In addition, the builtin-ChipScope debugger is connected through the serving PC and can be controlled and monitored.

The building blocks of the receiver are implemented on different FPGA resources as shown in Figure 18. The ADC-converted sample stream is down-converted and decimated to a baseband data rate. A single real analog signal is digitized to a $160 \mathrm{Msamples} / \mathrm{s}$ stream, which is the input to FPGA 1. I/ Q-down conversion is performed from the $70 \mathrm{MHz}$ IF and the decimator chain converts from a constant $160 \mathrm{Msamples/s} \mathrm{input} \mathrm{rate} \mathrm{down} \mathrm{to} 8 \mathrm{Msamples/s} \mathrm{I/Q-signal.} \mathrm{A} \mathrm{coarse} \mathrm{frequency}$ tuning (the quadricorrelator) is implemented by using a squared-root raised cosine (SRRC) filter and its derivative (dSRRC). Concerning the timing recovery, a Gardner timing error detector [18] and a Farrow interpolator adjust the correct symbol rate and phase. Correlation peaks computed on consecutive superframes by the frame synchronization algorithm (the NC-PDI, [32]) are used for a coarse timing synchronization and to fine-tune the Gardner algorithm. Once the start of the superframe is found and the residual timing error is sufficiently small, the symbol stream is descrambled and the fine frequency tuning [34] is applied to the symbol stream. Afterwards, channel 


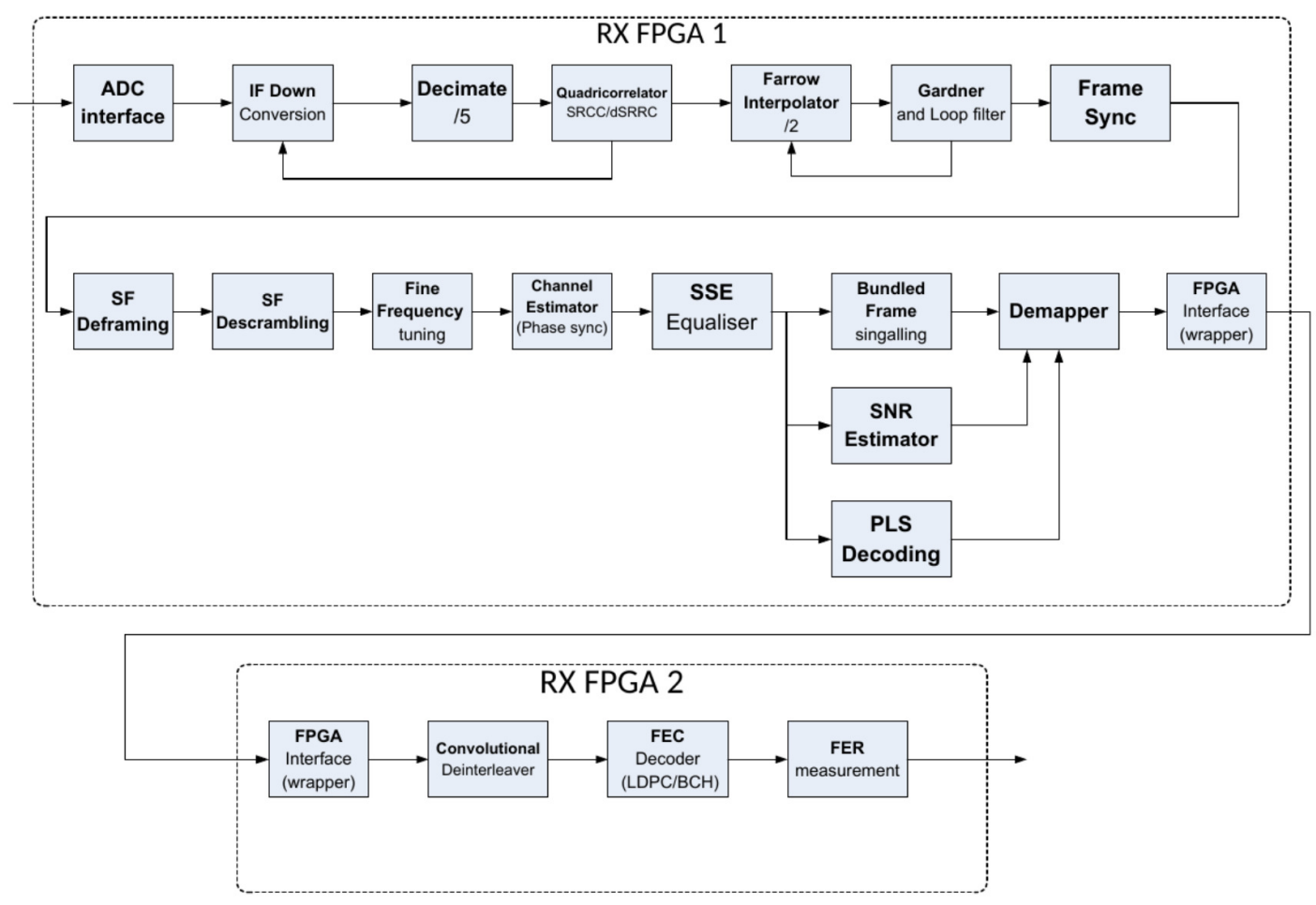

Figure 18. Detailed receiver block scheme.

estimation can be performed on the pilot fields. The symbol-spaced equalizer (SSE) removes the intersymbol interference from the symbol stream. From the equalized symbol stream, the physical laywer signalling code is detected, the bundled frames are marked in the stream, and the SNR is estimated. The calculated noise variance, received data symbol vectors, and the channel estimate matrices are input to the detector to produce soft LLRs of the stream. The block then forwards FEC frames to the COMMON processor for deinterleaving and decoding. The COMMON processing deinterleaves and decodes the LLRs. Deinterleaving includes the DVB-S2X specified deinterleaver and a placeholder for the convolutional channel deinterleaver. The convolutional interleaving can be included or excluded from the dataflow by control register. The decoded FEC words are then checked against reference data and a FER measurement is produced. The received data rate is estimated by calculating the number of received bytes per second.

5.2.3. Channel Firmware The considered impairments and their implementation are reported in Table II. The IZT C3040 satellite link emulator is used to model the satellite impairments. The Propsim channel emulator is used to model the actual radio channel and some other impairments as shown in Table II. Some terminal impairments are modeled inside the actual firmware by setting parameters off from their correct values. 
Table II. Hardware Impairments and Channel Effects.

\begin{tabular}{|c|c|c|c|}
\hline Impairment & Satellite Emulator & Radio Emulator & SDR \\
\hline IMUX & $\mathrm{X}$ & & \\
TWTA & $\mathrm{X}$ & & \\
OMUX & $\mathrm{X}$ & & \\
phase noise & $\mathrm{X}$ & & \\
\hline AWGN & & $\mathrm{X}$ & \\
CCI and ACI & & $\mathrm{X}$ & \\
Doppler effects & & $\mathrm{X}$ & \\
fading & & $\mathrm{X}$ & \\
shadowing & & $\mathrm{X}$ & \\
\hline carrier frequency offset & & & $\mathrm{X}$ \\
timing error & & & $\mathrm{X}$ \\
I/Q imbalance & & & \\
\hline
\end{tabular}

\section{PERFORMANCE ANALYSIS}

The purpose of this section is twofold. First, we provide a selection of simulation results with ideal assumptions aiming at assessing the performance of the SPD alone. Then, global results are shown, providing a realistic performance assessment of the whole communication system. All the results have been obtained by means of software simulations and with the hardware demonstrator.

\subsection{Predistortion}

The impact of SPD has been assessed by means of software simulations. The signal $s(t)$ in (4) has been formed by generating a random sequence of $3 \cdot 10^{5}$ symbols, while the output of the transponder $\tilde{y}(t)$ in (3) has been generated by the transit of $s(t)$ through IMUX, TWTA, and OMUX. The AM / AM and AM / PM characteristics of the TWTA, as well as the impulse responses of the IMUX and OMUX filters, are assumed to be known. In a practical context, they can be provided by the transponder manufacturer or by the operator before the launch of the satellite. In this numerical analysis, the recommended characteristics in [10] have been used (in particular, the conventional characteristics for the TWTA, and the filter responses for $36 \mathrm{MHz}$ bandwidth for IMUX and OMUX). Since SPD coefficients computed assuming a specific modulation format are valid for that modulation format only, this exercise was repeated for all the considered modulation formats (i.e., QPSK, 8PSK, and 16APSK). Further, since the coefficients are computed offline, no feedback from the user terminal is required.

The performance of the SPD has been assessed by considering two figures of merit: the achievable spectral efficiency (ASE) and the coded bit error rate (BER). The ASE $\eta$ can be computed (in $\mathrm{bit} / \mathrm{s} / \mathrm{Hz}$ ) as

$$
\eta=\frac{I(X ; Y)}{B T}
$$

where $I(X ; Y)$ is the mutual information between the transmitted symbol $X$ and the received symbol $Y, B$ is occupied bandwidth, and $T$ is the symbol period. It is a practical figure of merit 
since it does not require any encoding/decoding, assumes perfect synchronization, and provides an upper bound to the system spectral efficiency. However, gain predictions obtained in terms of ASE do not guarantee that the same gains could be obtained with the FEC codes prescribed by the standard. In order to assess the practical attainability of the predicted gains, coded BER curves have been computed by using the MODCODs in [1] and [2].

For the sake of generality, the investigated system considers a multicarrier scenario with three orthogonal carriers, with baudrate equal to $6 \mathrm{Mbaud}$ and roll-off 0.1 , IBO equal to $6 \mathrm{~dB}$, and a transponder with a $-3 \mathrm{~dB}$-bandwidth equal to $38 \mathrm{MHz}$. The SPD is applied to the aggregate signal, i.e., the multicarrier signal. This particular set-up was chosen in order to be representative of a generic feeder link gateway, rather than the specific hardware demonstrator described in Section 5. Further assumptions include perfect synchronization and absence of mobility. Figure 19 reports the ASE obtained for QPSK, 8PSK, and 16APSK. Since an exhaustive assessment of all the MODCODs of the standards would be out of scope, only few MODCODs have been tested as an example. In particular, Figure 20 reports the results for MODCODs 18 and 19 from DVB-S2 (i.e., 16APSK with code rates $2 / 3$ and $3 / 4$, respectively) and MODCODs 162, 166, and 168 from DVB-S2X (i.e., 16APSK with code rates $23 / 36,25 / 36$, and $13 / 18$, respectively).

The results shown in Figure 19 indicate that there exist codes providing a gain around $0.5 \mathrm{~dB}$ when the SPD is used. Moreover, the results reported in Figure 20 show that SPD provides a gain ranging approximately from $0.4 \mathrm{~dB}$ to $0.5 \mathrm{~dB}$ with practical MODCODs, which is in line with what predicted by the ASE curves. In other words, the MODCODs defined by the standards can be used with SPD without any code redesign.

It is worth mentioning that these gains are dependent on the considered scenario: symbol constellation, baudrate, roll-off, filters responses, AM / AM and AM / PM characteristics, IBO, and the receiver design as well (namely, the equalizer), they all have a non-negligible impact on the SPD design and, therefore, on its final performance. With so many degrees of freedom, an exhaustive investigation of the ultimate SPD performance becomes a formidable task, out of the scope of this paper.

\subsection{End-to-End Simulations and Tests}

In this section, results obtained by means of software full-fledged simulations are reported along with hardware measurements. Unlike the system described in Section 6.1, the simulation set-up considered for the end-to-end simulations is consistent with the hardware demonstrator described in Section 5, so as to make fair comparisons between software and hardware results. In order to span a wide range of realistic operational conditions, a set of test cases has been identified. Each test case represents a specific scenario (e.g., VL-SNR regime, pedestrian user, vehicular user, maritime environment). The presented investigation covers the whole forward link, from $\mathrm{BCH}$ encoder to BCH decoder. The adopted IMUX and OMUX characteristics are based on the one recommended in [10], with bandwidths equal to $36 \mathrm{MHz}$. Realistic channel and hardware impairments have been set separately for each test case, and the parameters characterizing the synchronization chain have been tuned accordingly. In particular, a typical suburban scenario was considered for LMSS. The assumption of usage of a highly directional antenna allowed to simplify the channel model from a Loo model [36] to a Ricean model. For MMSS, the considered scenario is characterized by a high elevation angle of the satellite and by a receive antenna with high directivity. Also, we abstract the 


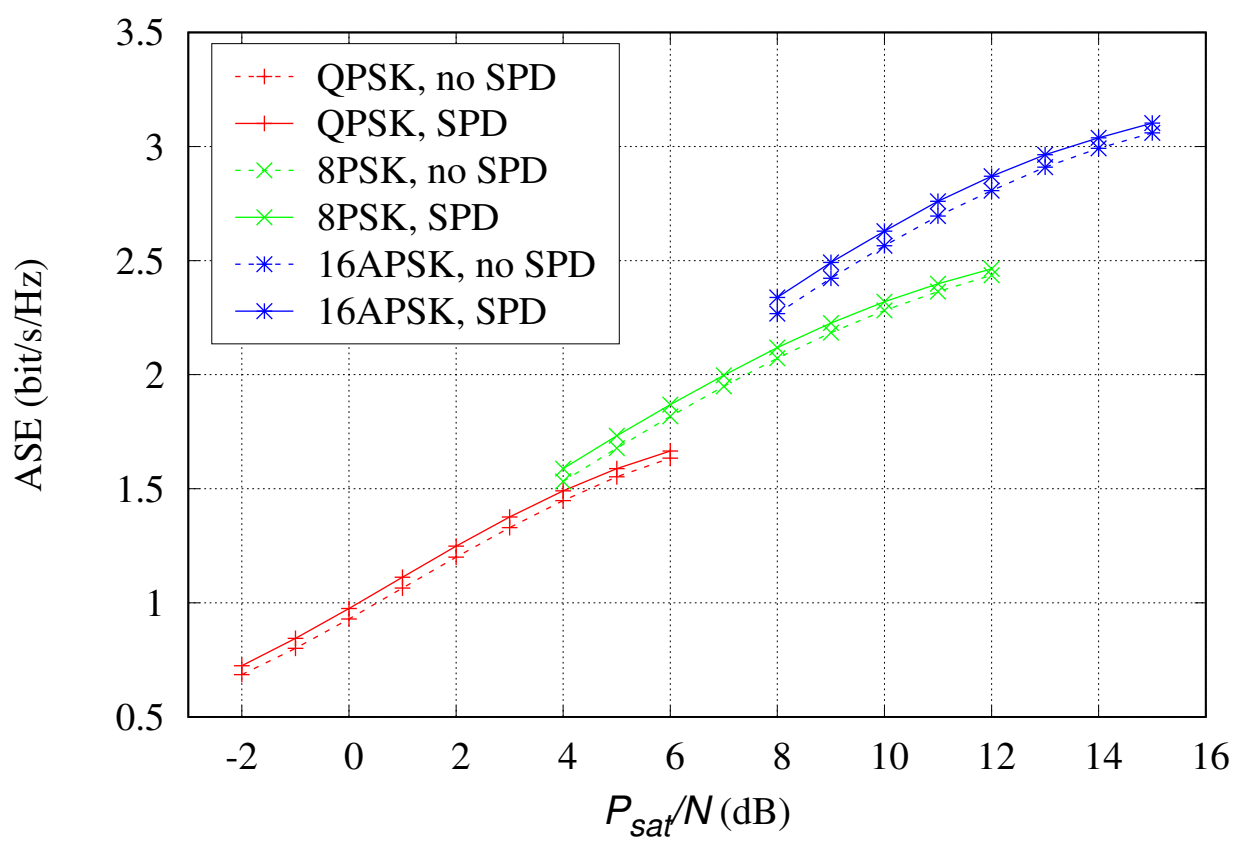

Figure 19. ASE for QPSK, 8PSK, and 16APSK with and without SPD.

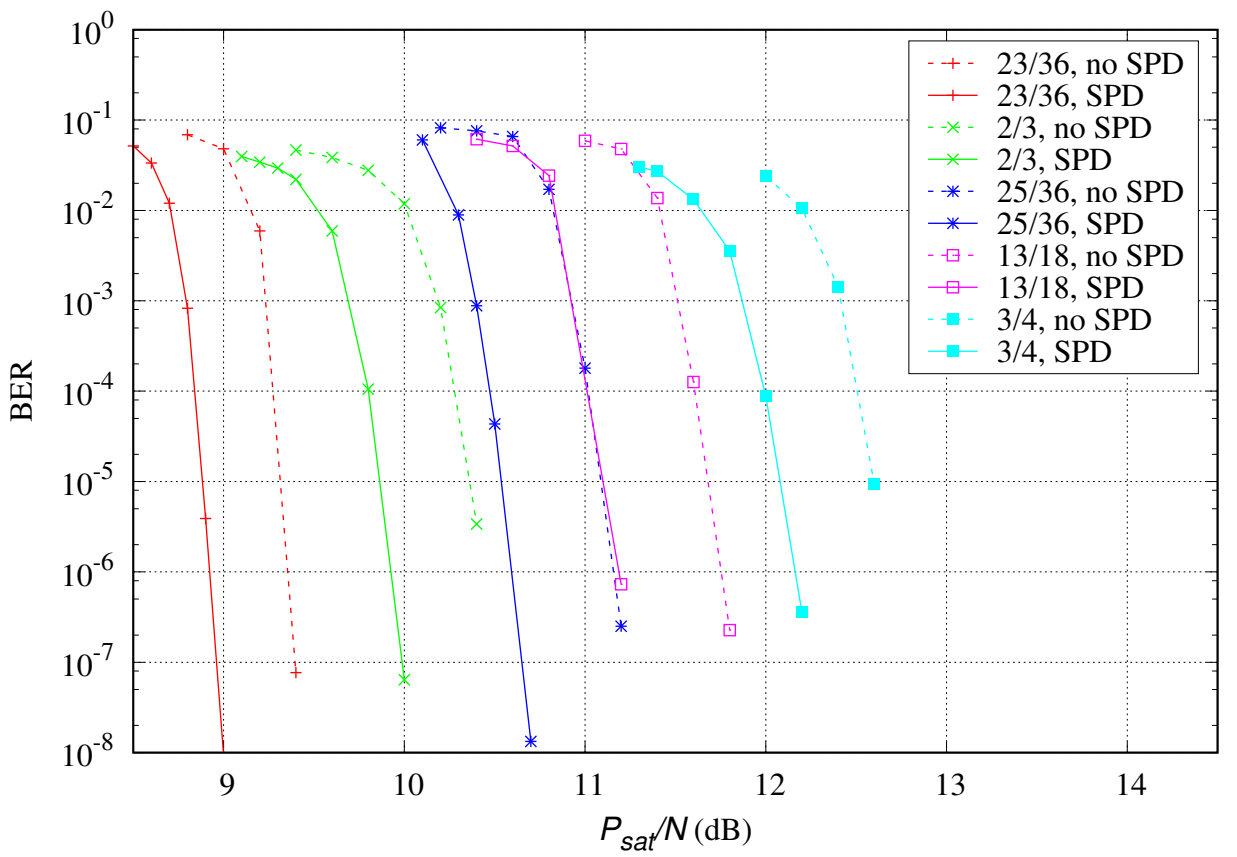

Figure 20. Coded BER for 16APSK MODCODs with and without SPD.

effects of the Ka-band (for VSAT test cases) and the L-band (for LMSS and MMSS test cases) by properly setting the parameters characterizing the channel.

SPD and the channel interleaver have been included when deemed necessary. Namely, SPD is not included in test cases using BPSK and QPSK, while the channel interleaver is adopted only 
Table III. Common Test Case Parameters.

\begin{tabular}{|c|c|}
\hline PARAMETER & VALUE \\
\hline timing drift (ppm) & 3 (0 for VSAT) \\
I/ Q phase imb. (deg) & \pm 3 \\
I/ Q amplit. imb. (dB) & \pm 0.6 \\
quadricorrelator loop bandwidth (normalized to $\left.R_{S}\right)$ & $2 \cdot 10^{-7}$ \\
NC-PDI correlation threshold & 1.55 \\
NC-PDI correlation length (symbols) & 5 \\
NC-PDI number of pilots & 720 \\
Gardner damping factor & 1.2 \\
Gardner detection gain & 3 \\
SSE number of taps & 9 \\
SSE training length (symbols) & 720 \\
TX oversampling factor & 10 \\
RX oversampling factor & 4 \\
\hline
\end{tabular}

when the user terminal is mobile (i.e., in LMSS and MMSS scenarios). Due to constraints on the considered hardware platform, the impact of the channel interleaver on the system performance has been assessed only via software simulation. All simulations assume an adaptive symbol-spaced equalizer (SSE) at the receiver: at the beginning of each simulation (in both software and hardware), the SSE is trained by a sequence of dummy superframes with a static realization of the channel (in particular, 10 superframes were used). During the performance assessment, the mobile channel evolves freely and the SSE tracks the channel variations by exploiting the pilots provided by the superframe structure. In particular, the training length used for updating the taps corresponds to 720 pilot symbols. In order to represent the different processing capabilities of the transmitter (i.e., gateway) and the receiver (i.e., user terminal), different oversampling factors have been used. As figure of merit, the FER has been considered for the validation of both software and hardware over the AWGN channel [2], and for the performance assessment.

The values taken by all the parameters (characterizing transmitter, channel, and receiver) for each test case are reported in Table III (parameters common to all the scenarios) and Tables IVVI (scenario-specific parameters). Parameters relative to the Gardner algorithm are defined in [37]. For all the test cases, a single carrier is considered and superframe format 3 is applied. The values chosen for the timing drift assume the usage of a very stable clock on both ends (e.g., by using the global navigation satellite system). However, the same synchronization chain can be used when a more severe timing drift is present, as shown for some VSAT test cases in Section 6.2.1. For VSAT, the phase noise is generated according to the phase noise mask reported in [10] (namely, mask P2 in Table 14), while for LMSS and MMSS the adopted phase noise mask for the L-band is reported in [38] (namely, in Table 5.5). In the following, the SNR is defined as $E_{s} / N_{0}$, where $E_{s}$ is the average symbol energy and $N_{0}$ the power spectral density of the thermal noise. 
Table IV. Test Case Parameters for VSAT.

\begin{tabular}{|c|c|c|c|c|c|c|}
\hline TEST CASE & V1 & V2 & V3 & V4 & V5 & V6 \\
\hline MODCOD & BPSK-1/5 & QPSK-4/15 & 8PSK-2/3 & 16APSK-8/9 & 8PSK-2/3 & 16 APSK-8/9 \\
$R_{S}$ (Mbaud) & 27.5 & 27.5 & 27.5 & 27.5 & 33 & 33 \\
roll-off & 0.2 & 0.2 & 0.2 & 0.2 & 0.1 & 0.1 \\
IBO (dB) & 3 & 1 & 3 & 5 & 3 & 9 \\
freq. err. (\% of $\left.R_{S}\right)$ & 10 & 10 & 10 & 10 & 10 & yes \\
SPD & no & no & yes & yes & yes \\
$\begin{array}{c}\text { Gardner loop bandwidth } \\
\text { (normalized to } R_{S} \text { ) }\end{array}$ & $2 \cdot 10^{-5}$ & $2 \cdot 10^{-5}$ & $2 \cdot 10^{-5}$ & $2 \cdot 10^{-5}$ & $2 \cdot 10^{-5}$ & $2 \cdot 10^{-5}$ \\
\hline
\end{tabular}

Table V. Test Case Parameters for LMSS.

\begin{tabular}{|c|c|c|c|c|}
\hline TEST CASE & L1 & L2 & L3 & L4 \\
\hline MODCOD & QPSK-4/15 & QPSK-4/15 & 8 PSK-2/3 & 16 APSK-8/9 \\
$R_{S}$ (Mbaud) & 4 & 4 & 4 & 4 \\
roll-off & 0.1 & 0.1 & 0.1 & 0.1 \\
IBO (dB) & 1 & 1 & 3 & 9 \\
user speed (km/h) & 3 & 50 & 3 & 50 \\
freq. err. (\% of $\left.R_{S}\right)$ & 1 & 1 & 1 & 1 \\
SPD & no & no & yes & yes \\
Gardner loop bandwidth (normalized to $\left.R_{S}\right)$ & $4 \cdot 10^{-5}$ & $5 \cdot 10^{-5}$ & $6 \cdot 10^{-5}$ & $5 \cdot 10^{-5}$ \\
$K$-factor (dB) & 10 & 10 & 10 & 10 \\
\hline
\end{tabular}

Table VI. Test Case Parameters for MMSS.

\begin{tabular}{|c|c|c|c|c|}
\hline TEST CASE & M1 & M2 & M3 & M4 \\
\hline MODCOD & BPSK-1/5 & QPSK-4/15 & 8 PSK-2/3 & 16 APSK-8/9 \\
$R_{S}$ (Mbaud) & 4 & 4 & 4 & 4 \\
roll-off & 0.2 & 0.2 & 0.2 & 0.2 \\
IBO (dB) & 3 & 1 & 3 & 9 \\
user speed (km/h) & 25 & 25 & 25 & 25 \\
freq. err. (\% of $\left.R_{S}\right)$ & 1 & 1 & 1 & 1 \\
SPD & no & no & yes & yes \\
Gardner loop bandwidth (normalized to $\left.R_{S}\right)$ & $4 \cdot 10^{-5}$ & $5 \cdot 10^{-5}$ & $6 \cdot 10^{-5}$ & $5 \cdot 10^{-5}$ \\
$K$-factor $(\mathrm{dB})$ & 17 & 17 & 17 & 17 \\
\hline
\end{tabular}

6.2.1. VSAT Outbound FER results for test cases V1 (i.e., BPSK in VL-SNR regime) and V2 (QPSK) are presented in Figure 21. Compared to the AWGN channel, the system performance (in software) over the realistic satellite channel shows a loss lower than $0.2 \mathrm{~dB}$ for both the test 
cases. The performance in hardware shows a loss around $0.2 \mathrm{~dB}$ (at FER $=10^{-5}$ ) with respect to the AWGN channel for V1, and around $0.7 \mathrm{~dB}$ for $\mathrm{V} 2$. Even though there is no timing drift assumed for the VSAT scenarios (as reported in Table III), if a timing drift equal to $100 \mathrm{ppm}$ of the baudrate $R_{S}$ is added, Figure 21 shows that a negligible loss occurs in the V1 scenario. The reported results confirm that reliable communication is possible in the VL-SNR regime. Also, in Figure 21 it can be seen that, for FER $<5 \cdot 10^{-5}$, the hardware implementation of V1 outperforms the corresponding software implementation. The cause of such behavior is purely computational: the last point of the software curve has been computed using very few wrong frames (due to an imposed limitation to the available simulation time), which makes the FER value itself less reliable than the others. On the other hand, measurements in hardware were much faster, and the reported results at very low FER are more reliable than the corresponding ones in software.

Figure 22 shows similar results for test cases V3 and V5 (8PSK). For both test cases the hardware shows a loss around $0.3 \mathrm{~dB}$ with respect to the corresponding software performance. Comparing the curves across the test cases (i.e., software with software and hardware with hardware), a loss around $0.2 \mathrm{~dB}$ can be seen. This loss is caused by the more challenging operational conditions of V5, namely the smaller roll-off (which makes the synchronization more difficult) and the higher baudrate (which increases the intersymbol interference introduced by the transponder filters). Moreover, the figure illustrates the impact of the SSE, which provides a gain approximately equal to $1 \mathrm{~dB}$ in software.

FER results for test cases V4 and V6 (16APSK) are shown in Figure 23. The hardware performance is again in line with the software results, but in this case a loss of around $0.5 \mathrm{~dB}$ can be seen by comparing hardware and software curves for V4. The figure also reports a further cross-check on the impact of the SPD. The gain reported in Figure 23 is higher than the ones in Figure 20 because scenario V4 is more challenging than the one considered for the SPD assessment (namely, single carrier with a higher baudrate and a lower IBO). Further, by increasing the timing drift in the hardware to $100 \mathrm{ppm}$, a negligible loss occurs, showing that the synchronization chain properly compensates for the impairments. For V6 the hardware performance is in agreement with the software results. Again, by increasing the timing drift to $100 \mathrm{ppm}$, a negligible loss (i.e., lower than $0.1 \mathrm{~dB}$ ) can be seen.

6.2.2. LMSS Scenario The FER results for the LMSS scenarios L1 and L2 (QPSK) are presented in Figure 24, showing that with both pedestrian (L1) and vehicular (L2) mobility the channel interleaver is greatly beneficial. The hardware performance without channel interleaver is also shown to be in agreement with the corresponding software results for both test cases. Also, the higher the terminal speed, the more challenging is the scenario, and therefore the worse is the performance. The same qualitative behavior can be seen is Figure 25 for test cases L3 (8PSK) and L4 (16APSK), where the channel interleaver is shown to improve the performance in software, and the hardware results agree with the software ones without interleaver.

6.2.3. MMSS Scenario As for the LMSS scenario, the results for MMSS reported in Figure 26 and Figure 27 show that software and hardware results (without the channel interleaver) are consistent. Again, the interleaver has a large impact (a gain between 2 and $3 \mathrm{~dB}$ at FER $=10^{-4}$ ). The reference curves in black in both figures were obtained by using the channel model for the VSAT scenario, and the system parameters specified in Table VI (except the user speed and the $K$-factor). 


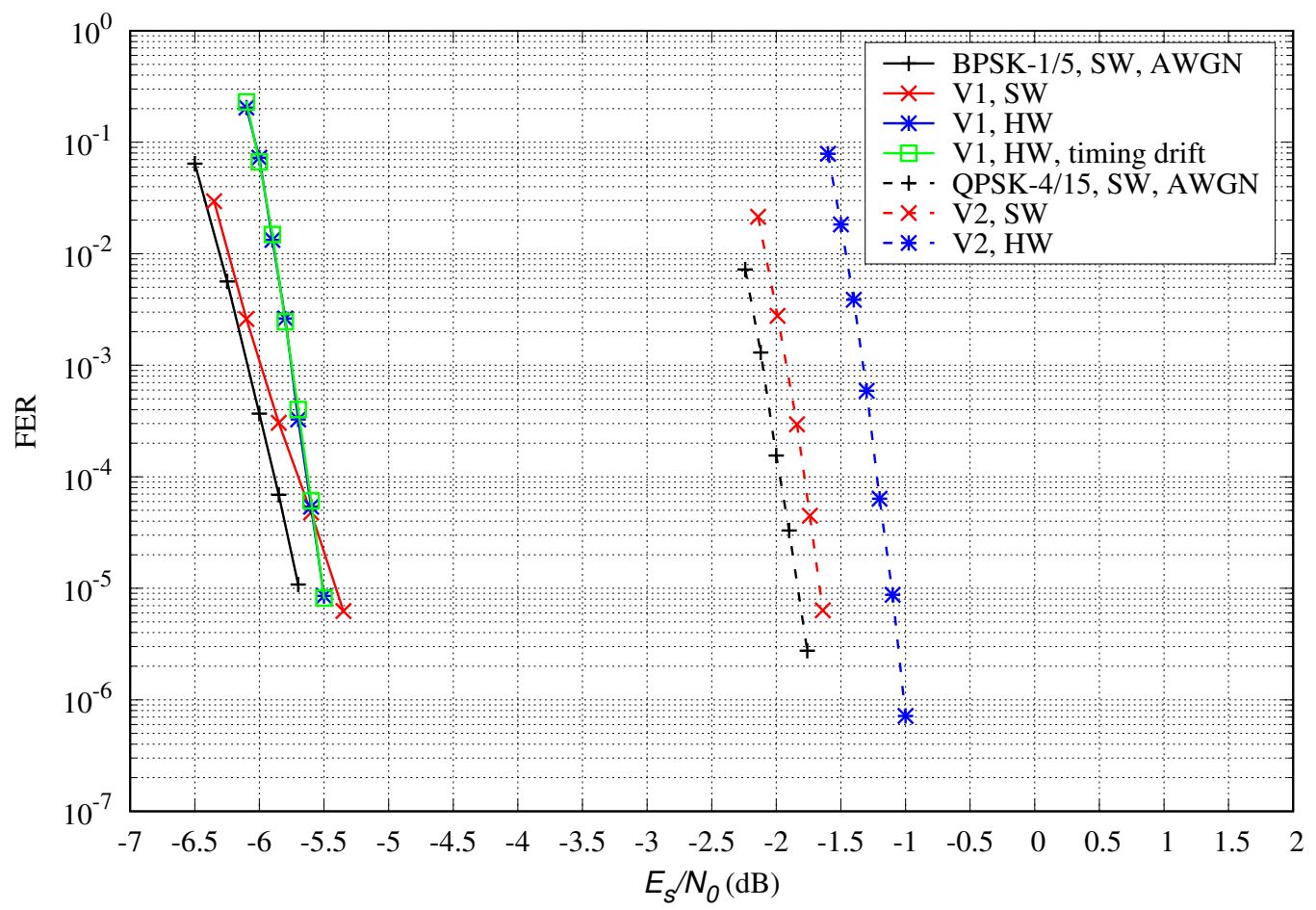

Figure 21. FER curves for test cases V1 and V2.

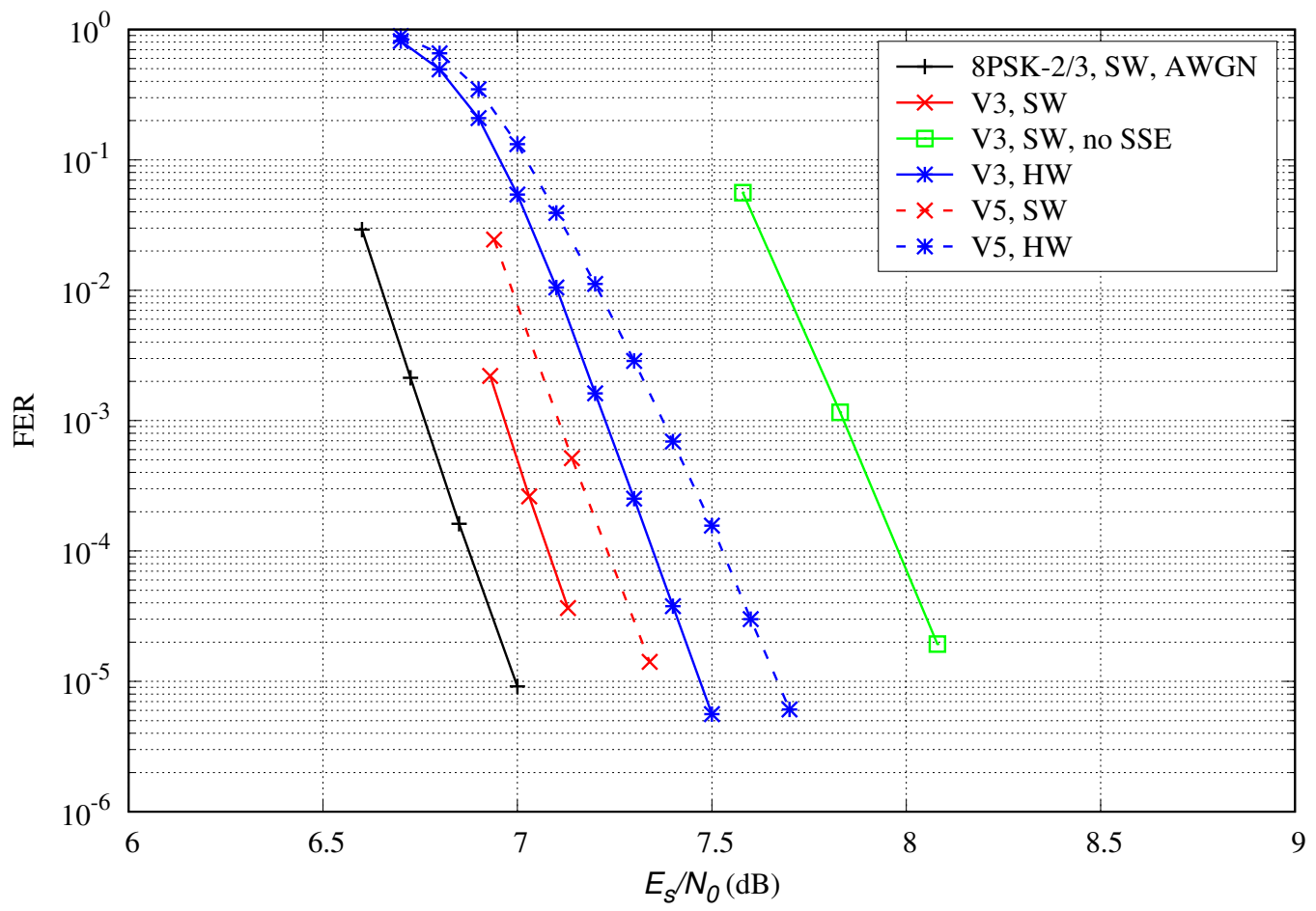

Figure 22. FER curves for test cases V3 and V5. 


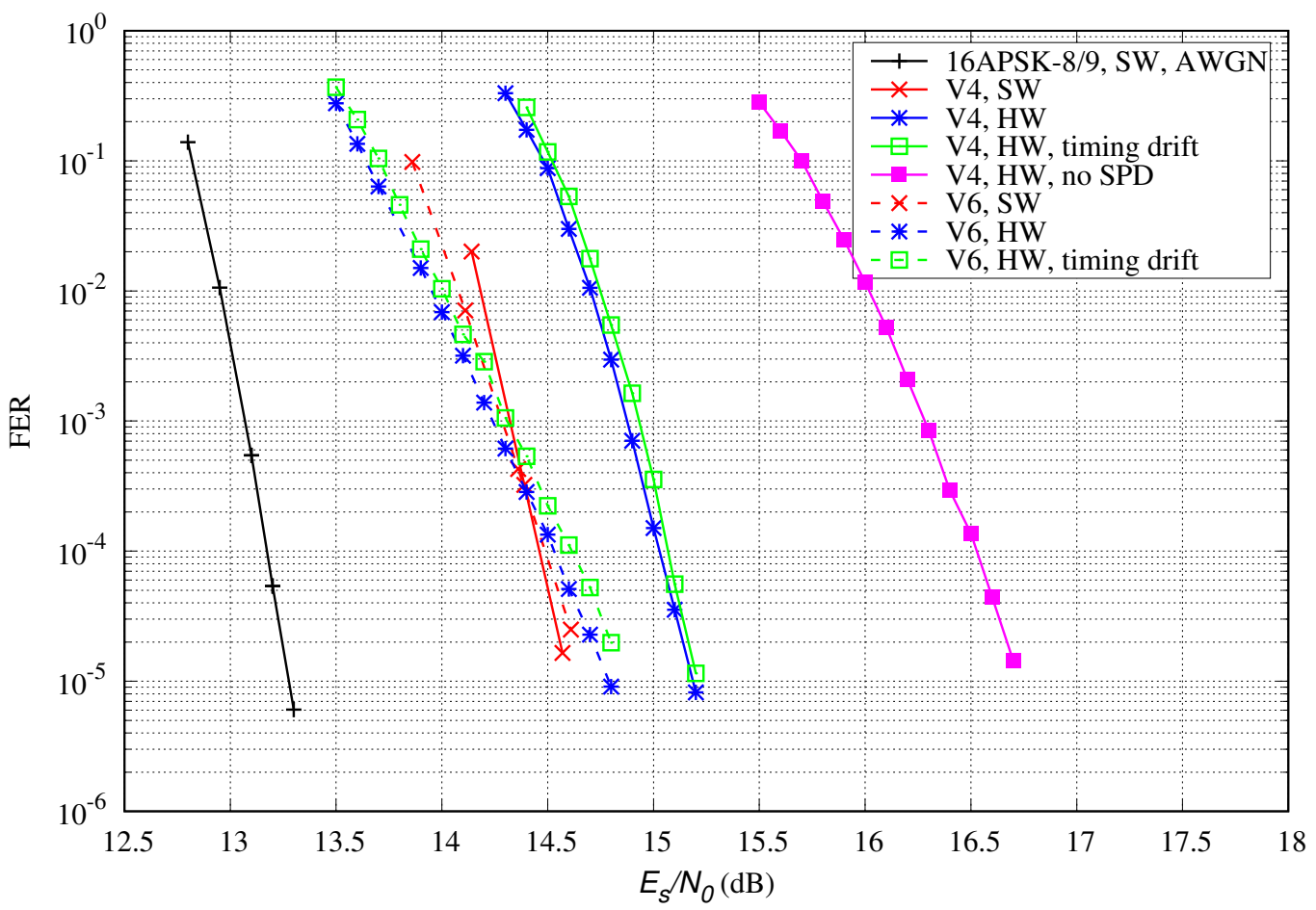

Figure 23. FER curves for test cases V4 and V6.

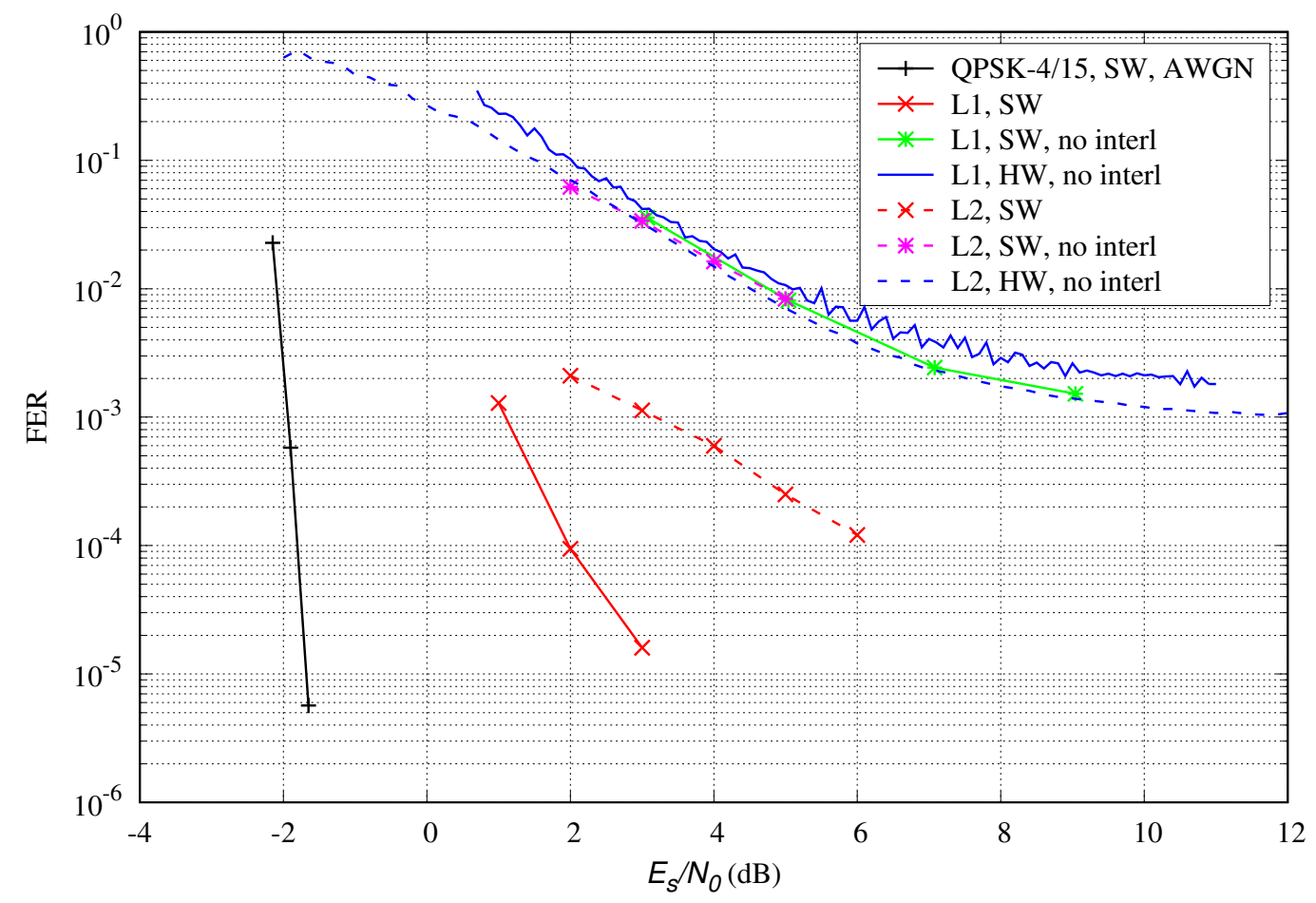

Figure 24. FER curves for test cases L1 and L2. 


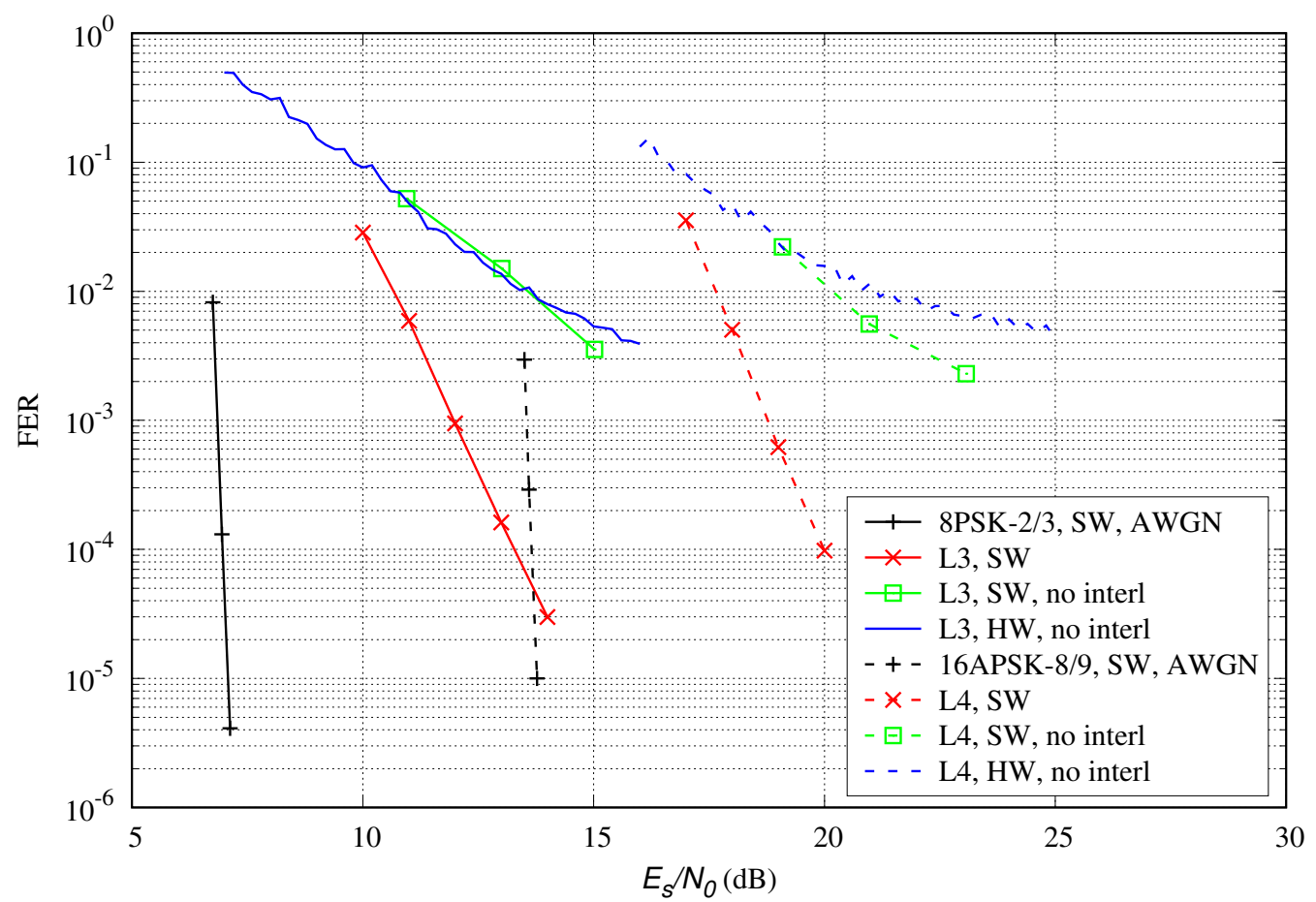

Figure 25. FER curves for test cases L3 and L4.

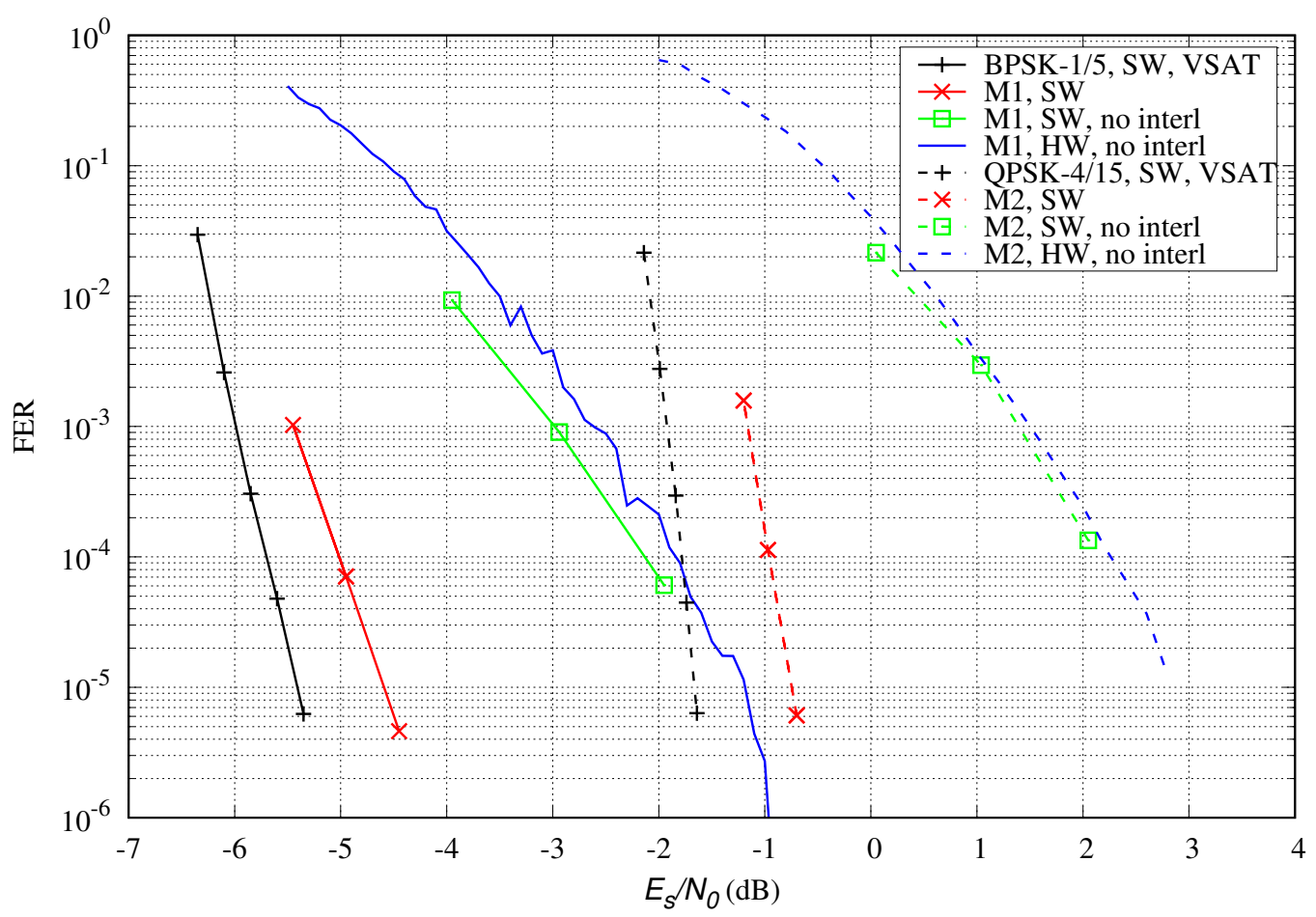

Figure 26. FER curves for test cases M1 and M2. 


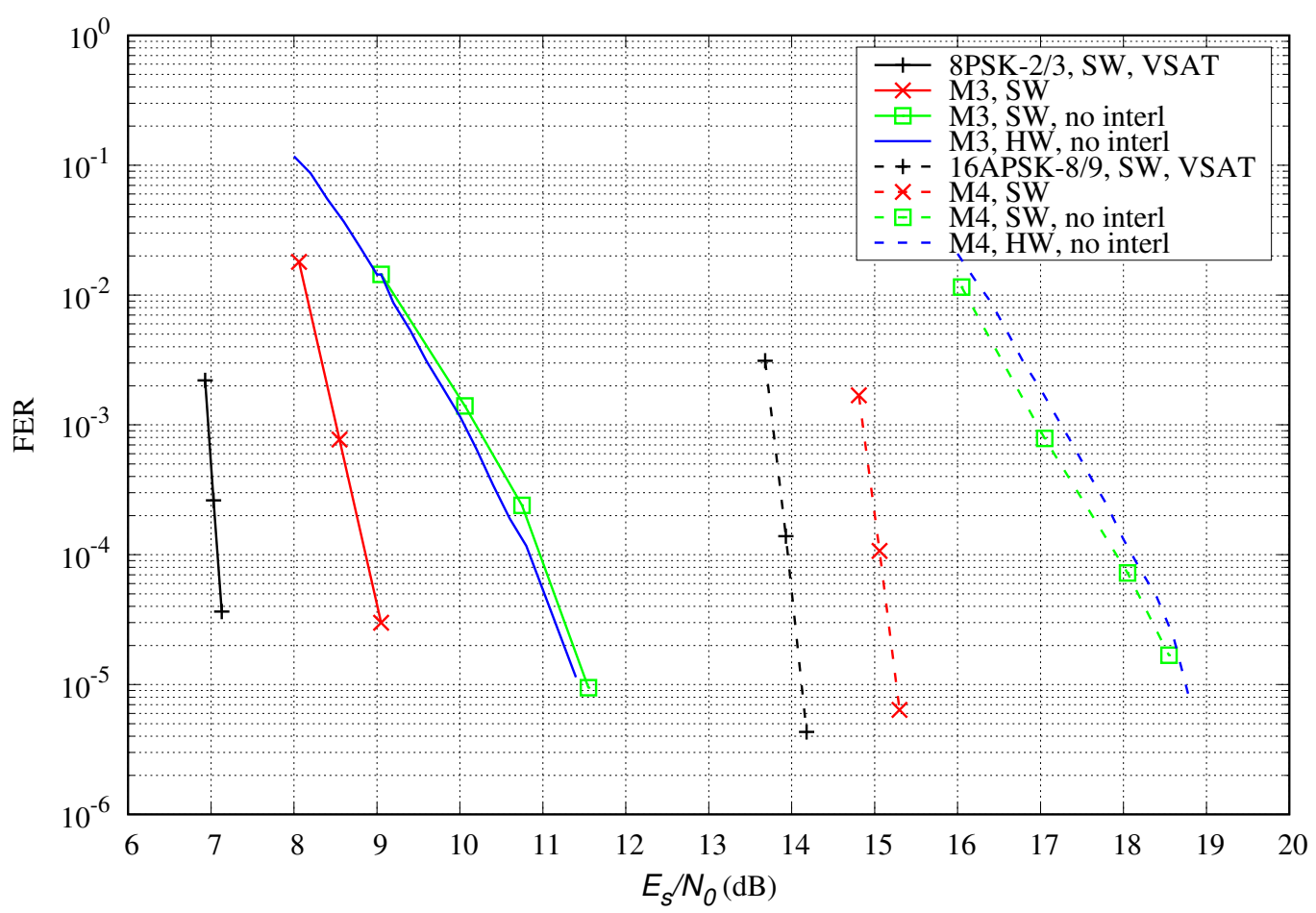

Figure 27. FER curves for test cases M3 and M4.

\section{CONCLUSIONS}

In this paper, we have shown that the superframing structure, provided in the Annex E of the DVBS2X standard, can be a common technology enabler for different mobile scenarios like land-mobile, aeronautical, and maritime, in addition to fixed scenarios such as VSAT. Moreover, the superframing structure was actively exploited by the channel interleaver and by a robust synchronization chain to simplify the implementation and improve the performance. A SPD algorithm and the channel interleaver were adopted as key PHY techniques to cope with the transponder nonlinearity and the channel fading. As a result, reliable communication was attained in the VL-SNR regime and in mobile scenarios. A hardware testbed incorporating the PHY techniques with the DVB-S2X superframing format 3 was devised (the first, at the best of the authors' knowledge, to assess the performance of superframing in mobile scenarios), and its functionalities were validated by comparing the measured performance against the ones obtained by means of software simulations.

\section{ACKNOWLEDGEMENTS}

This work was carried out in a European Space Agency project, contract no.4000110120/14/NL/NR [39]. The authors would like to thank Juha Korpi who previously worked at VTT, Juha Ylitalo from Bittium, and Ilias Panagiotopoulos from ESA for the precious help. Opinions, interpretations, recommendations and conclusions presented in this paper are those of the authors and are not necessarily endorsed by the European Space Agency. 


\section{REFERENCES}

1. ETSI EN 302 307-1, V1.4.1, "Digital Video Broadcasting (DVB); Second generation framing structure, channel coding and modulation systems for Broadcasting, Interactive Services, News Gathering and other broadband satellite applications; Part 1: DVB-S2", Nov. 2014.

2. ETSI EN 302 307-2, V1.1.1, "Digital Video Broadcasting (DVB); Second generation framing structure, channel coding and modulation systems for Broadcasting, Interactive Services, News Gathering and other broadband satellite applications; Part 2: DVB-S2 Extensions", Oct. 2014.

3. Willems K., "DVB-S2X Demystified", white paper by Newtec, March 2014.

4. ETSI EN 302583 V1.2.1, "Digital Video Broadcasting (DVB); Framing Structure, channel coding and modulation for Satellite Services to Handheld devices (SH) below 3 GHz", Dec. 2011.

5. "Report of the TM-S2 Study Mission on green field technologies for satellite transmissions", DVB white paper, March 2014.

6. Rohde C., Alagha N., De Gaudenzi R., Stadali H., and Mocker G., "Super-framing: a powerful physical layer frame structure for next generation satellite broadband systems", Int. J. Satell. Commun. and Network., vol. 34., no. 3, pp. 413-438, 2016.

7. Arapoglu P.-D., Ginesi A., Cioni S., Erl S., Clazzer F., Andrenacci S., and Vanelli-Coralli A., "DVB-S2X-enabled precoding for high throughput satellite systems", Int. J. Satell. Commun. and Network., vol. 34., no. 3, pp. 439-455, 2016.

8. "White Paper on the use of DVB-S2X for DTH applications, DSNG \& Professional Services, Broadband Interactive Services and VL-SNR applications", DVB Document A172, March 2015.

9. Höyhtyä M., Huusko J., Kiviranta M., Solberg K., and Rokka J., "Connectivity for Autonomous Ships: Architecture, Use Cases, and Research Challenges", in Proc. IEEE Int. Conf. on ICT Convergence (ICTC), 2017.

10. ETSI TR 102 376-2, V1.1.1, "Digital Video Broadcasting (DVB); Implementation guidelines for the second generation systems for Broadcasting, Interactive Services, News Gathering and other broadband satellite applications; Part 2: S2 Extensions (DVB-S2X)", Nov. 2015.

11. ITU-R P.618-12 Recommendation, "Propagation data and prediction methods required for the design of Earth-space telecommunication systems", P Series, Radiowave Propagation, July 2015.

12. ITU-R P.1511-1 Recommendation, "Topography for Earth-space propagation modelling", P Series, Radiowave Propagation, July 2015.

13. ITU-R P.681-9 Recommendation, "Propagation data required for the design of Earth-space land mobile telecommunication systems", P Series, Radiowave Propagation, Sep. 2016.

14. ITU-R P.531-13 Recommendation: "Ionospheric propagation data and prediction methods required for the design of satellite services and systems", P Series, Radiowave Propagation, Sep. 2016.

15. ETSI TR 125 996, V14.0.0, "Universal Mobile Telecommunications System (UMTS); Spatial channel model for Multiple Input Multiple Output (MIMO) simulations (3GPP TR 25.996)", April 2017.

16. ETSI TS 102 584, V1.3.1, "Digital Video Broadcasting (DVB); DVB-SH Implementation Guidelines", Nov. 2011.

17. Corazza G. E., Digital Satellite Communications, Springer Ed., 2007.

18. Mengali U. and D’Andrea A. N., Synchronization Techniques for Digital Receivers, Plenum, 1997.

19. Lee L.-N., Eroz M., and Becker N., "Modulation, coding, and synchronization for mobile and very small satellite terminals, as part of the updated DVB-S2 standard", Int. J. Satell. Commun. and Network., vol. 34, no. 3, pp. 377-386, 2016.

20. Hekkala A., Hiivala M., Lasanen M., Perttu J., Vieira L. C., Gomes N. J., and Nkansah A., "Predistortion of radio over fiber links: algorithms, implementation, and measurements", IEEE Trans. on Cir. and Sys. I: Regular Papers, vol. 59, no. 3, pp. 664-672, 2012.

21. Casini E., De Gaudenzi R., and Ginesi A., "DVB-S2 modem algorithms design and performance over typical satellite channels", Int. J. Satell. Commun. and Network., vol. 22, pp. 281-318, May/June 2004.

22. Piazza R., Shankar M. R. B., and Ottersten B., "Non-parametric data predistortion for non-linear channels with memory", in Proc. IEEE Int. Conf. on Acoustics, Speech, and Signal Processing (ICASSP), 2013.

23. Piazza R., Shankar M. R. B., and Ottersten B., "Multicarrier LUT-based Data Predistortion for Non-linear Satellite Channels", in Proc. IEEE Int. Conf. on Comm. (ICC), 2014.

24. Eun C. and Powers E., "A new Volterra predistorter based on the indirect learning architecture", IEEE Trans. on Signal Proc., vol. 45, no. 1, pp. 223-227, Jan. 1997.

25. Mazzali N., Shankar M. R. B., and Ottersten B., "On-board signal predistortion for digital transparent satellites", IEEE 16th Int. Workshop on Signal Processing Advances in Wireless Communications (SPAWC), Stockholm, pp. 535-539, 2015. 
26. Zhou D. and DeBrunner V. E., "Novel Adaptive Nonlinear Predistorters Based on the Direct Learning Algorithm", IEEE Trans. Signal Proc., vol. 55, no. 1, pp. 120-133, Jan. 2007.

27. Biglieri E., Barberis S., and Catena M., "Analysis and compensation of nonlinearities in digital transmission systems", IEEE J. Select. Areas Commun., vol. 6, no. 1, pp. 42-51, Jan. 1988.

28. Yedidia J. S., "Message-Passing Algorithms for Inference and Optimization", J. Stat. Phys., pp. 860-890, 2011.

29. Johnson S .G., The NLopt nonlinear-optimization package, http://ab-initio.mit.edu/nlopt.

30. Powell M. J. D., "A direct search optimization method that models the objective and constraint functions by linear interpolation", in Adv. in Optimization and Numerical Analysis, Springer Ed., p. 51-67, 1994.

31. D'Andrea A. N. and Mengali U., "Design of quadricorrelators for automatic frequency control systems", IEEE Trans. Commun., vol. 41, no. 6, pp. 988-997, June 1993.

32. Corazza G. E., Pedone R., and Villanti M., "Frame acquisition for continuous and discontinuous transmission in the forward link of satellite systems", Int. J. Satell. Commun. and Network., vol. 24, no. 2, pp. 185-201, 2006.

33. Viterbi A. J., CDMA: Principles of Spread Spectrum Communication, Addison-Wesley, 1995.

34. Mengali U. and Morelli M., "Data-aided frequency estimation for burst digital transmission", IEEE Trans. on Comm., vol. 45, pp. 23-25, Jan. 1997.

35. Gilchriest C. E., "Signal-to-Noise Monitoring", JPL Space Programs Summary no. 37-27, vol. IV, pp. 169-176, June 1966.

36. Loo C., "A statistical model for a land mobile satellite link", IEEE Trans. Veh. Tech., vol. 34, no. 3, pp. 122-127, Aug. 1985.

37. MathWorks, Documentation for MATLAB R2017b, 2017.

38. ETSI TS 102 744-2-2, V1.1.1, "Satellite Earth Stations and Systems (SES); Family SL Satellite Radio Interface (Release 1); Part 2: Physical Layer Specifications; Sub-part 2: Radio Transmission and Reception", Oct. 2015.

39. European Space Agency, "Novel Ground Components Prototype beyond DVB-S2 for Broadband Satellite Networks", information available at: https://artes.esa.int/projects/novel. 